\title{
Experimental and Theoretical Insights into the Electronic Properties of Anionic N-Heterocyclic Dicarbenes through the Rational Synthesis of Their Transition Metal Complexes
}

Alina A. Grineva, ${ }^{\dagger, \ddagger}$ Oleg A. Filippov,,$\stackrel{\text { }, \|}{ }$ Yves Canac, ${ }^{\dagger}$ Jean-Baptiste Sortais, ${ }^{\dagger, \neq}$ Sergei E. Nefedov, ${ }^{\ddagger}$ Noël Lugan, ${ }^{\dagger, \star}$ Vincent César ${ }^{\dagger, \star}$ and Dmitry A. Valyaev ${ }^{\dagger, *}$

† LCC-CNRS, Université de Toulouse, CNRS, UPS, 205 route de Narbonne, 31077 Toulouse Cedex 4, France $\ddagger$ Kurnakov Institute of General and Inorganic Chemistry, Russian Academy of Sciences, 31 Leninsky Pr., Moscow, 119991, Russia

$\S$ A. N. Nesmeyanov Institute of Organoelement Compounds (INEOS), Russian Academy of Sciences, 28 Vavilov str., GSP-1, B-334, Moscow, 119991, Russia

II Shemyakin-Ovchinnikov Institute of Bioorganic Chemistry, Russian Academy of Sciences, 16/10 MiklukhoMaklay Str., Moscow 117997, Russia

\# Institut Universitaire de France, 1 rue Descartes, 75231 Paris Cedex 05, France

\section{Supporting Information}




\section{Table of Contents}

Figures S1-S2. NMR spectra of complex $\mathrm{CpMn}(\mathrm{CO})_{2}(\mu$-dlMes $) \mathrm{Fe}(\mathrm{CO})_{2} \mathrm{Cp}(3)$

Figures S3-S4. NMR spectra of complex [CpFe(CO) ${ }_{2}$ (alMes)](OTf) ([4](OTf))

S5-6

Figures S5-S6. NMR spectra of complex $\left[\mathrm{CpFe}(\mathrm{CO})_{2}\left(\mu\right.\right.$-dIMes) $\left.\mathrm{Fe}(\mathrm{CO})_{2} \mathrm{Cp}\right](\mathrm{OTf})([5](\mathrm{OTf}))$

S7-8

Figures S7-S8. NMR spectra of complex $\left[\mathrm{CpFe}(\mathrm{CO})_{2}(\mathrm{IMes})\right](\mathrm{OTf})([6](\mathrm{OTf}))$

S9-10

2. Electrochemical studies of $\mathrm{Mn}(\mathrm{I})$ and $\mathrm{Fe}(\mathrm{II}) \mathrm{NHC}$ complexes

S11

Figure S9. Cyclic voltammogram of complex $\mathrm{CpMn}(\mathrm{CO})_{2}(\mathrm{IMes})(\mathbf{1})$

S11

Figure S10. Cyclic voltammogram of complex $\mathrm{CpMn}(\mathrm{CO})_{2}(\mu$-dIMes $) \mathrm{Fe}(\mathrm{CO})_{2} \mathrm{Cp}(3)$

S11

Figure S11. Cyclic voltammogram of complex [CpFe(CO) ${ }_{2}$ (alMes)](OTf) $([4](\mathrm{OTf}))$

S12

Figure S12. Cyclic voltammogram of complex $\left[\mathrm{CpFe}(\mathrm{CO})_{2}(\mu\right.$-dlMes $\left.) \mathrm{Fe}(\mathrm{CO})_{2} \mathrm{Cp}\right](\mathrm{OTf})([5](\mathrm{OTf}))$

S12

Figure S13. Cyclic voltammogram of complex $\left[\mathrm{CpFe}(\mathrm{CO})_{2}(\mathrm{IMes})\right](\mathrm{OTf})([6](\mathrm{OTf}))$

S13

\section{Computational results}

S14

Table S1. Key structural data of calculated $\mathrm{Mn}(\mathrm{I})$ and Fe(II) NHC complexes

S14

Table S2. Energy difference between the conformers $\mathrm{Mn}(\mathrm{I})$ and $\mathrm{Fe}(\mathrm{II}) \mathrm{NHC}$ complexes

S14

Table S3. Characteristics of bond critical points for $\pi(\mathrm{C}=\mathrm{C}) \ldots \pi^{*}(\mathrm{CO})$ interligand interactions in optimized $\mathrm{Mn}(\mathrm{I})$ and $\mathrm{Fe}(\mathrm{II}) \mathrm{NHC}$ complexes

S14

Figures S14-S18. AIM molecular graphs for $\mathrm{Mn}(\mathrm{I})$ and $\mathrm{Fe}(\mathrm{II}) \mathrm{NHC}$ complexes

$\mathrm{S} 15$

Table S4. Characteristics of bond critical points for metal-carbene bonds in optimized $\mathrm{Mn}(\mathrm{I})$ and $\mathrm{Fe}(\mathrm{II}) \mathrm{NHC}$ complexes

S17

Table S5. Selected AIM charges for optimized $\mathrm{Mn}(\mathrm{I})$ and $\mathrm{Fe}(\mathrm{II}) \mathrm{NHC}$ complexes

S17

Table S6. Selected NBO charges for optimized $\mathrm{Mn}(\mathrm{I})$ and $\mathrm{Fe}(\mathrm{II}) \mathrm{NHC}$ complexes

S17

Table S7. Calculated $v_{\text {co }}$ bands for optimized $\mathrm{Mn}(\mathrm{I})$ and $\mathrm{Fe}(\mathrm{II}) \mathrm{NHC}$ complexes

S18

Table S8. ETS-NOCV analysis data for metal-carbene bonding in optimized $\mathrm{Mn}(\mathrm{I})$

and $\mathrm{Fe}$ (II) NHC complexes bearing IMes, alMes and dIMes ligands

S18

Comparison of metal-carbon bonding between model $\sigma$-complexes $\mathrm{Cp}(\mathrm{CO})_{2} \mathrm{Fe}-\mathrm{R}$

(7-9) and Fe(II) IMes, alMes and dIMes complexes

S19

Figure S19. DFT optimized geometries for $\mathrm{Fe}(\mathrm{II})$ complexes $\mathrm{Cp}(\mathrm{CO})_{2} \mathrm{Fe}-\mathrm{R}$ (7-9)

S19

Table S9. Comparison of the selected characteristics of BCPs for Fe-C bonds in $\mathrm{Fe}(\mathrm{II})$

$\sigma$-complexes 7-9 with those of Fe-NHC bonds in IMes, alMes and dlMes complexes

S19

Table S10. The comparison of ETS-NOCV data for Fe-C bonding in optimized Fe(II)

$\sigma$-complexes 7-9 with those of Fe-NHC bonding in IMes, alMes and dIMes complexes

S20

Figures S20-S22. NOCV deformation electron density for model $\mathrm{Fe}(\mathrm{II}) \sigma$-complexes

$\mathrm{Cp}(\mathrm{CO})_{2} \mathrm{Fe}-\mathrm{R}$ 7-9

S21

Figure S23-S34. NOCV deformation electron density for $\mathrm{Mn}(\mathrm{I})$ and $\mathrm{Fe}(\mathrm{II}) \mathrm{NHC}$

complexes bearing IMes, alMes and dIMes ligands 
1. Copies of the NMR spectra for Mn(I) and Fe(II) NHC complexes

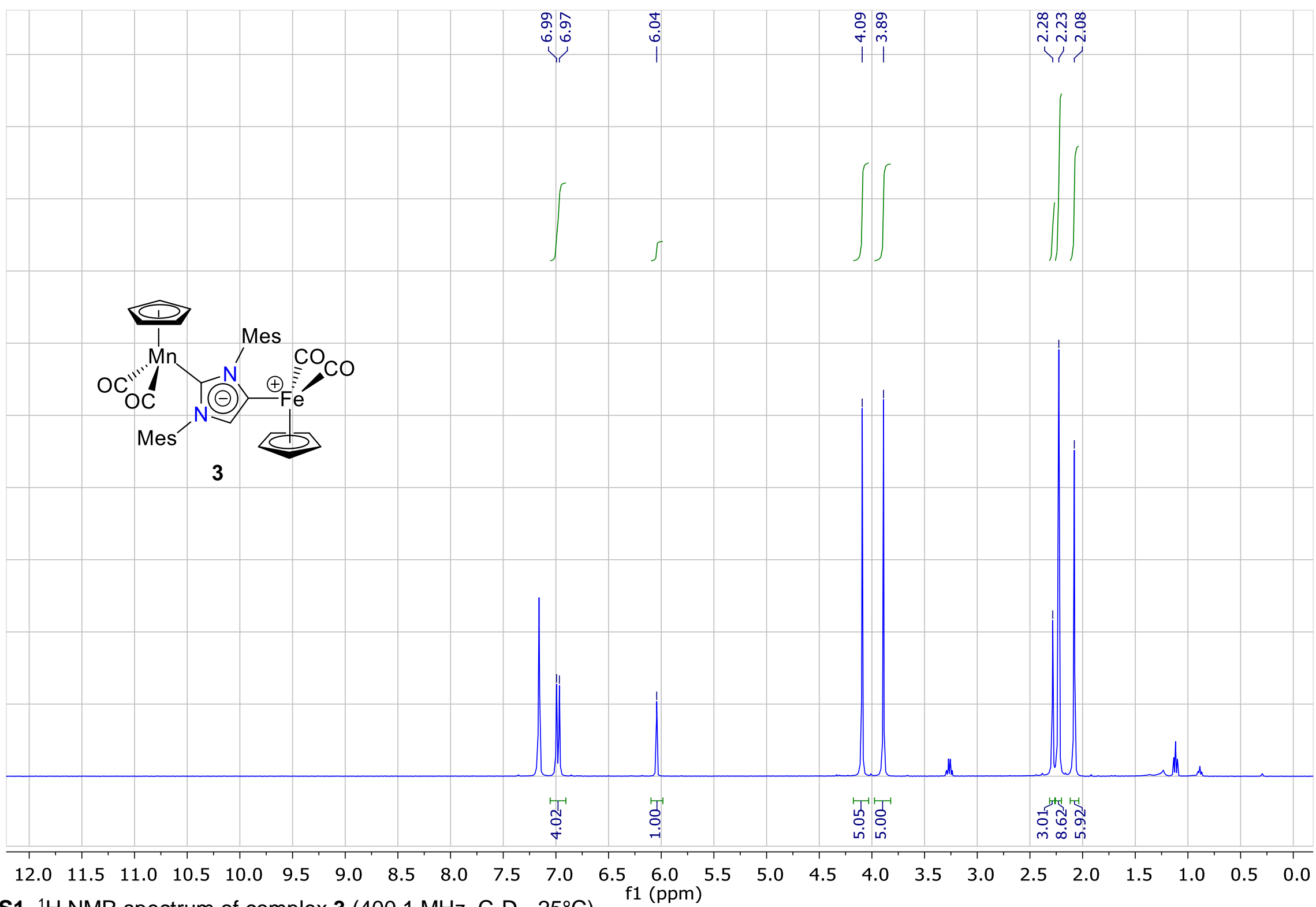

Figure S1. ${ }^{1} \mathrm{H}$ NMR spectrum of complex $3\left(400.1 \mathrm{MHz}, \mathrm{C}_{6} \mathrm{D}_{6}, 25^{\circ} \mathrm{C}\right)$ 


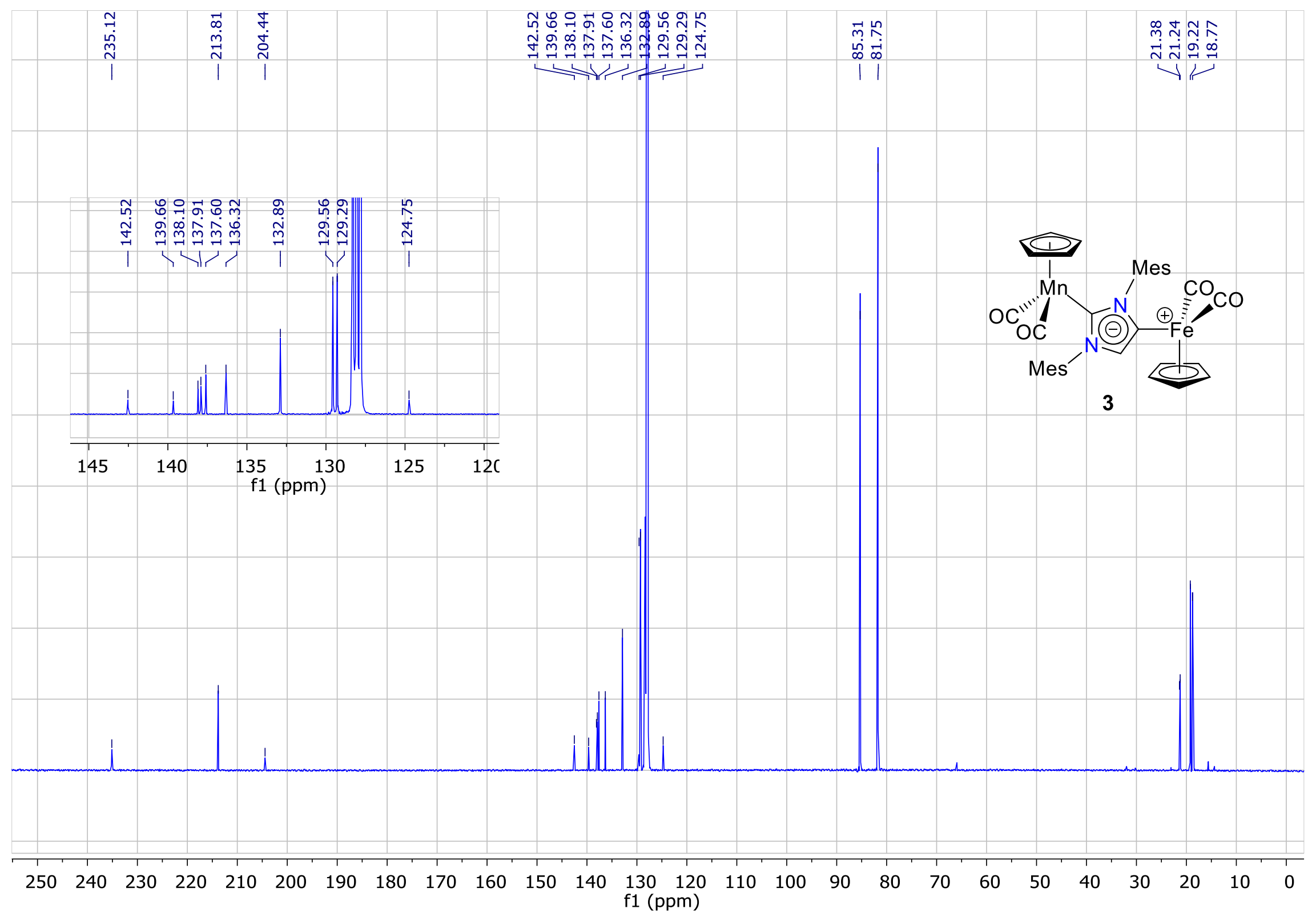

Figure S2. ${ }^{13} \mathrm{C}\left\{{ }^{1} \mathrm{H}\right\}$ NMR spectrum of complex $3\left(100.6 \mathrm{MHz}, \mathrm{C}_{6} \mathrm{D}_{6}, 25^{\circ} \mathrm{C}\right)$ 


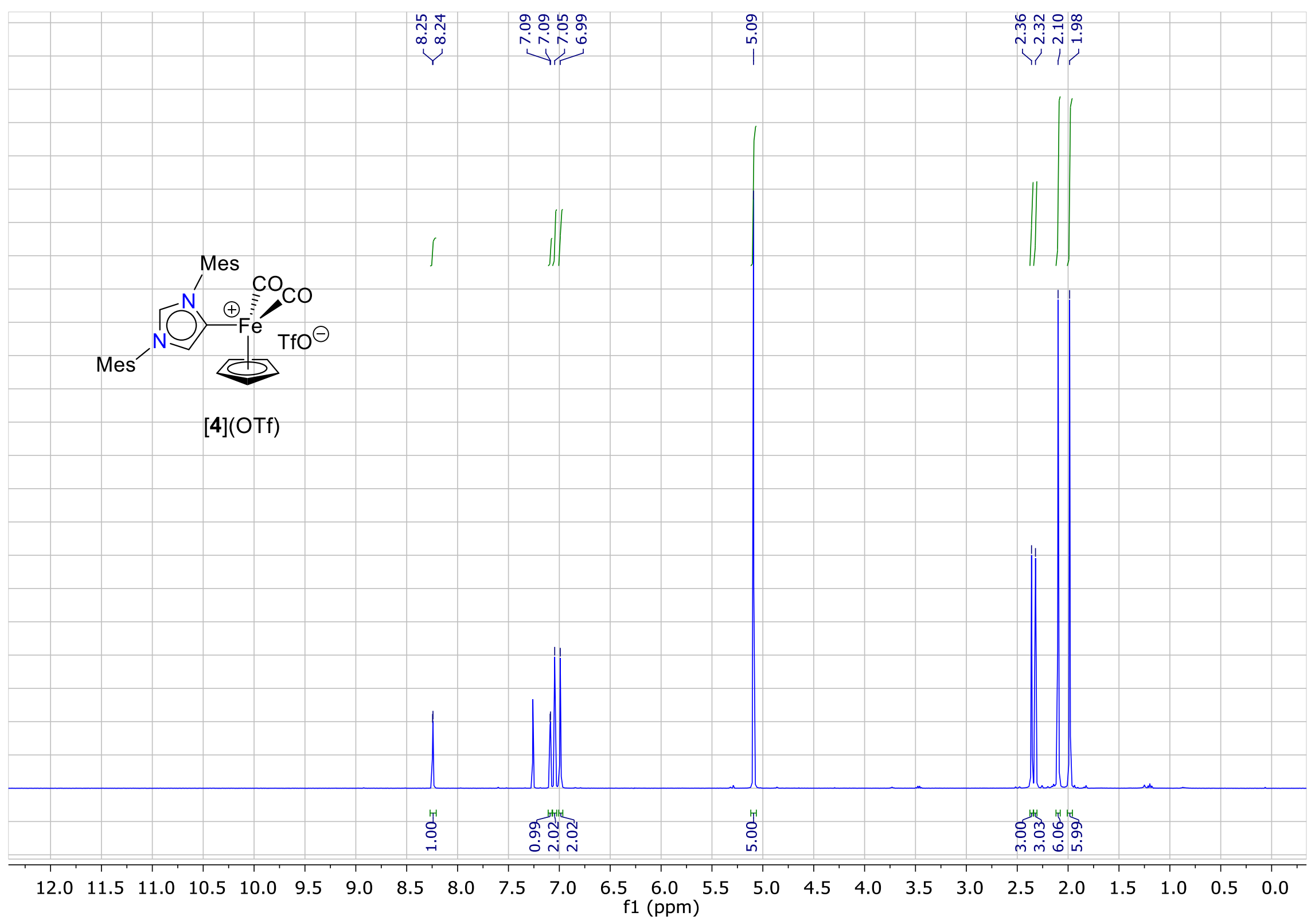

Figure S3. ${ }^{1} \mathrm{H}$ NMR spectrum of complex [4](OTf) $\left(400.1 \mathrm{MHz}, \mathrm{CDCl}_{3}, 2^{\circ} \mathrm{C}\right)$ 


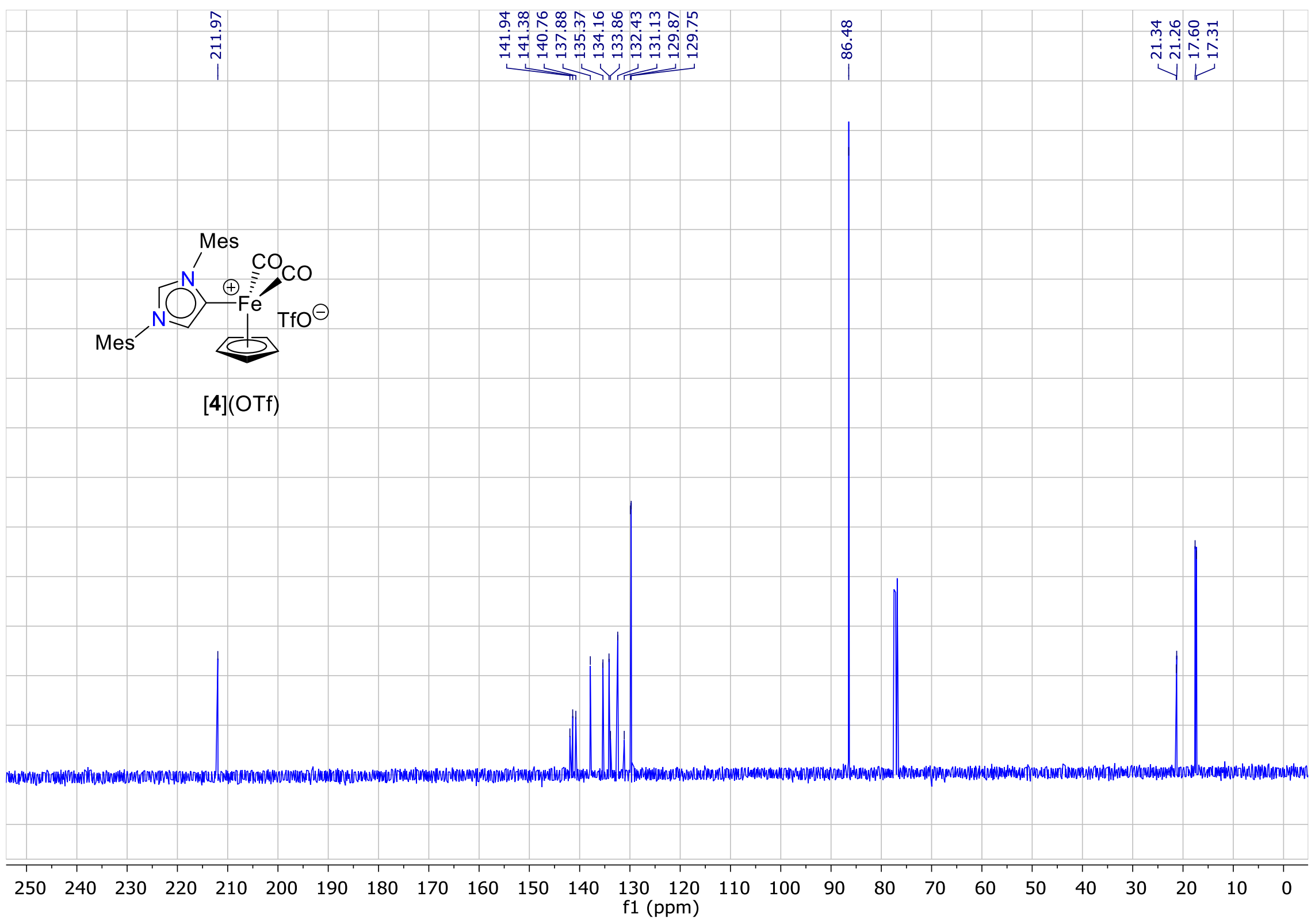

Figure S4. ${ }^{13} \mathrm{C}\left\{{ }^{1} \mathrm{H}\right\}$ NMR spectrum of complex [4](OTf) $\left(100.6 \mathrm{MHz}, \mathrm{CDCl}_{3}, 25^{\circ} \mathrm{C}\right)$ 


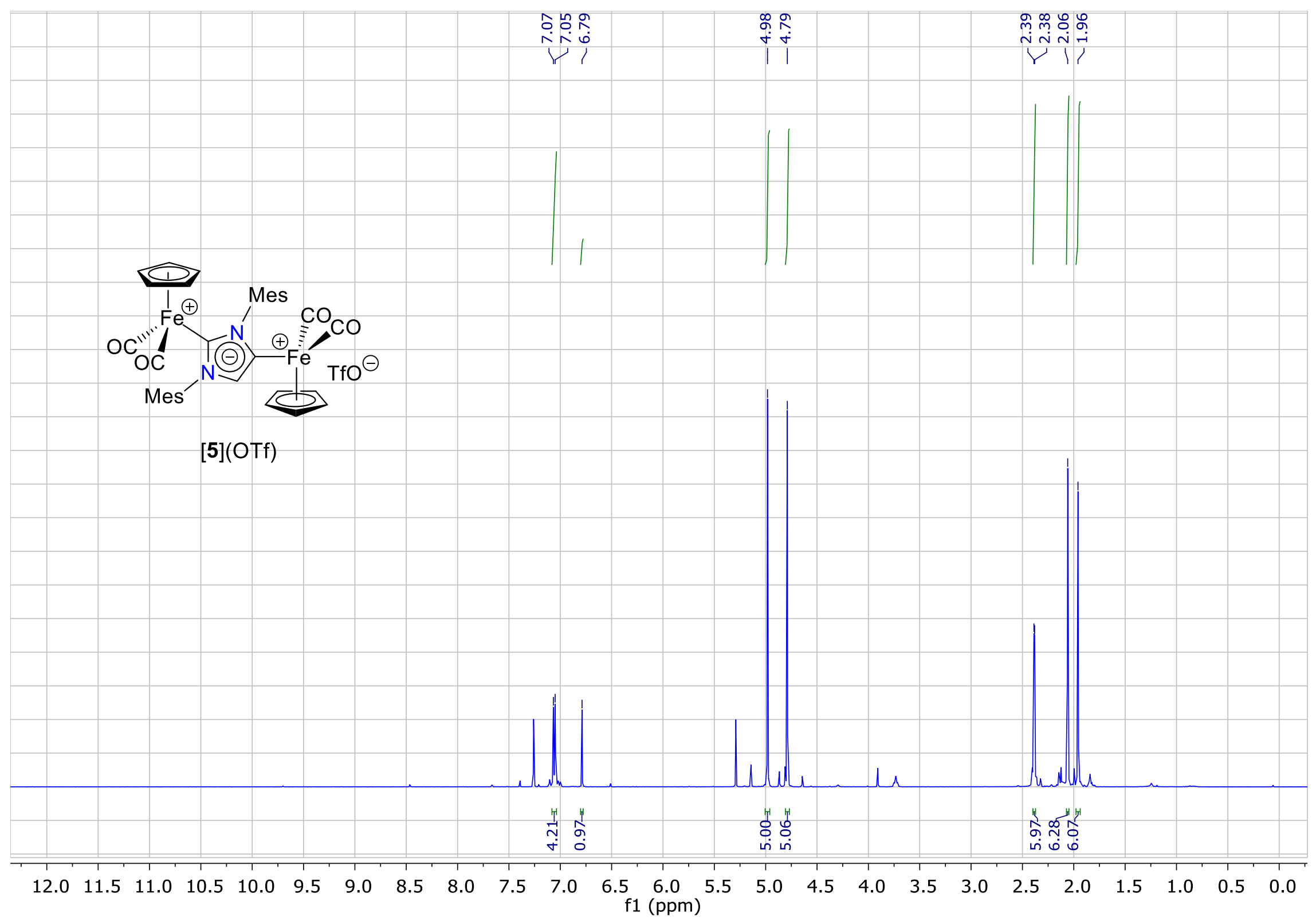

Figure S5. ${ }^{1} \mathrm{H}$ NMR spectrum of complex [5](OTf) $\left(400.1 \mathrm{MHz}, \mathrm{CDCl}_{3}, 25^{\circ} \mathrm{C}\right)$ 


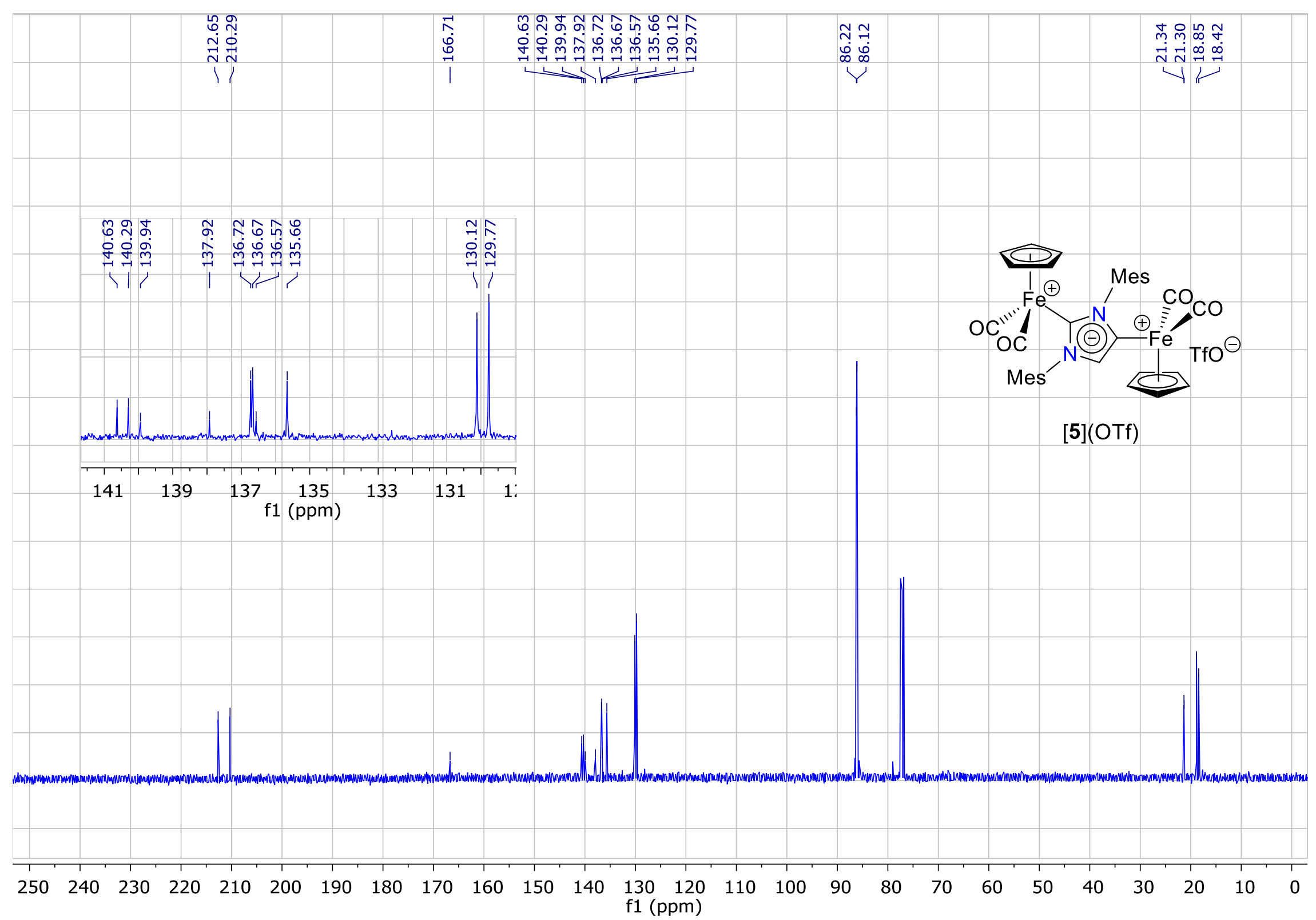

Figure S6. ${ }^{13} \mathrm{C}\left\{{ }^{1} \mathrm{H}\right\}$ NMR spectrum of complex [5](OTf) $\left(100.6 \mathrm{MHz}, \mathrm{CDCl}_{3}, 25^{\circ} \mathrm{C}\right)$ 


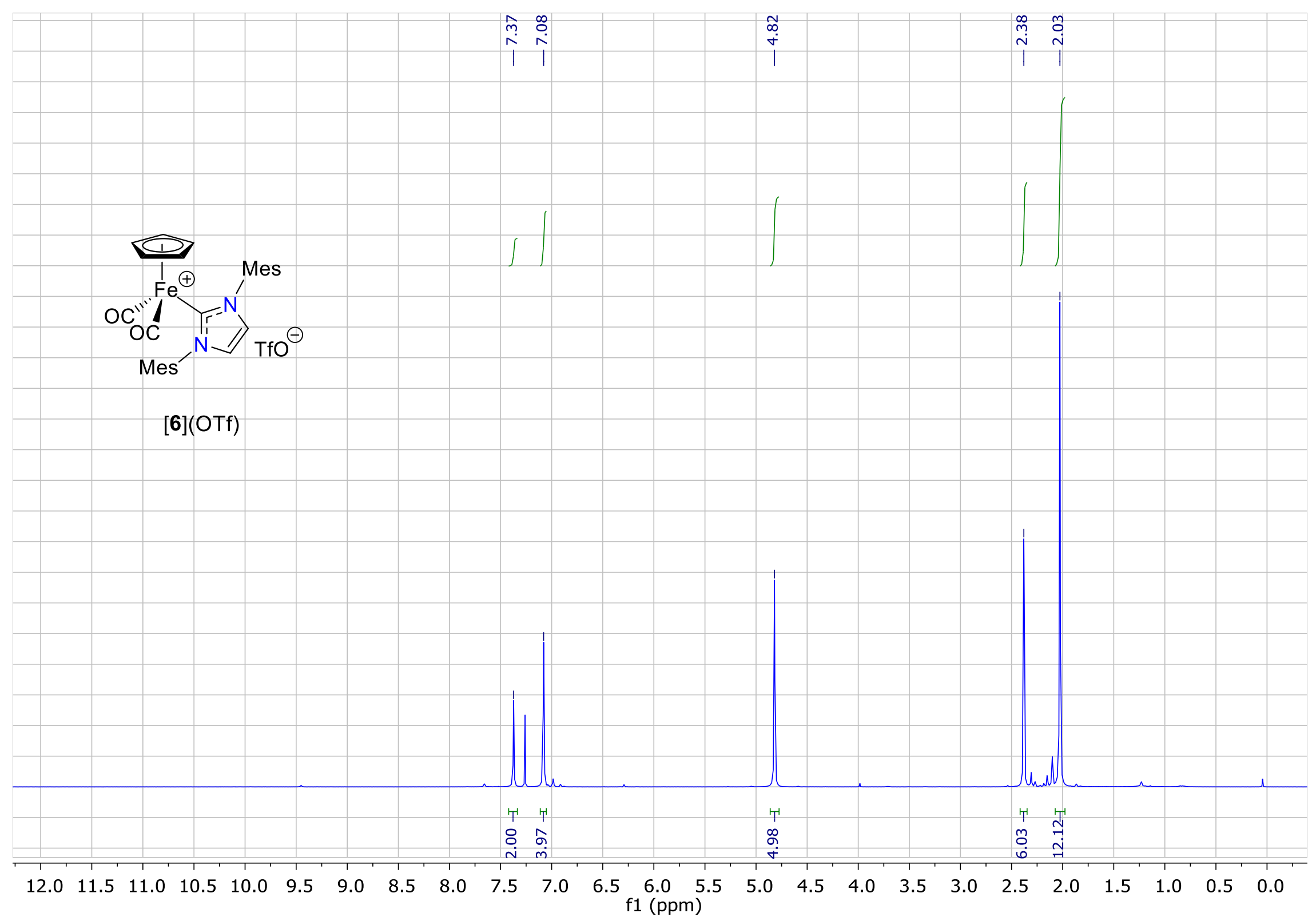

Figure S7. ${ }^{1} \mathrm{H}$ NMR spectrum of complex [6](OTf) $\left(400.1 \mathrm{MHz}, \mathrm{CDCl}_{3}, 25^{\circ} \mathrm{C}\right)$ 


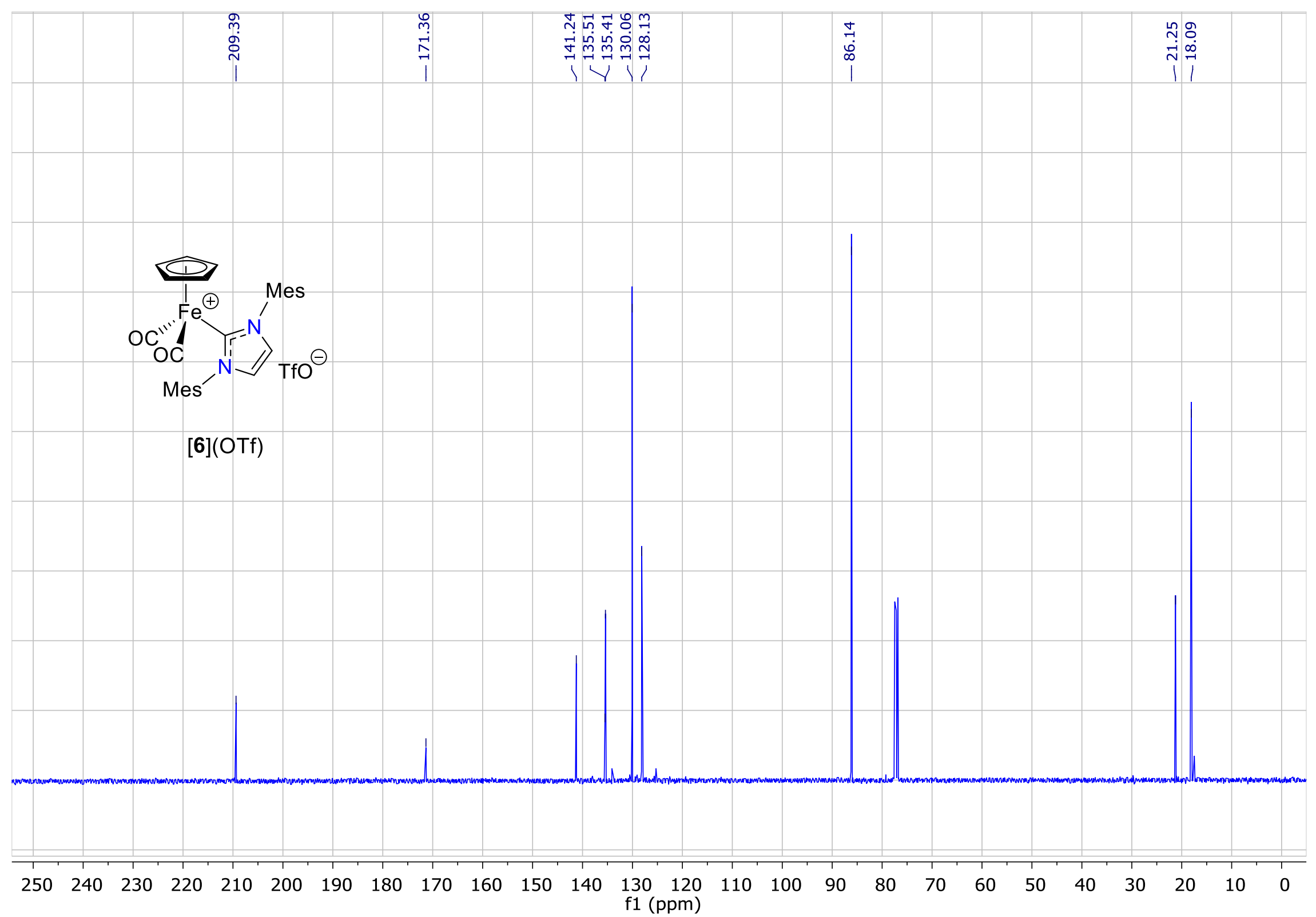

Figure S8. ${ }^{13} \mathrm{C}\left\{{ }^{1} \mathrm{H}\right\} \mathrm{NMR}$ spectrum of complex [6](OTf) $\left(100.6 \mathrm{MHz}, \mathrm{CDCl}_{3}, 25^{\circ} \mathrm{C}\right)$ 


\section{Electrochemical studies of $\mathrm{Mn}(\mathrm{I})$ and $\mathrm{Fe}(\mathrm{II}) \mathrm{NHC}$ complexes}

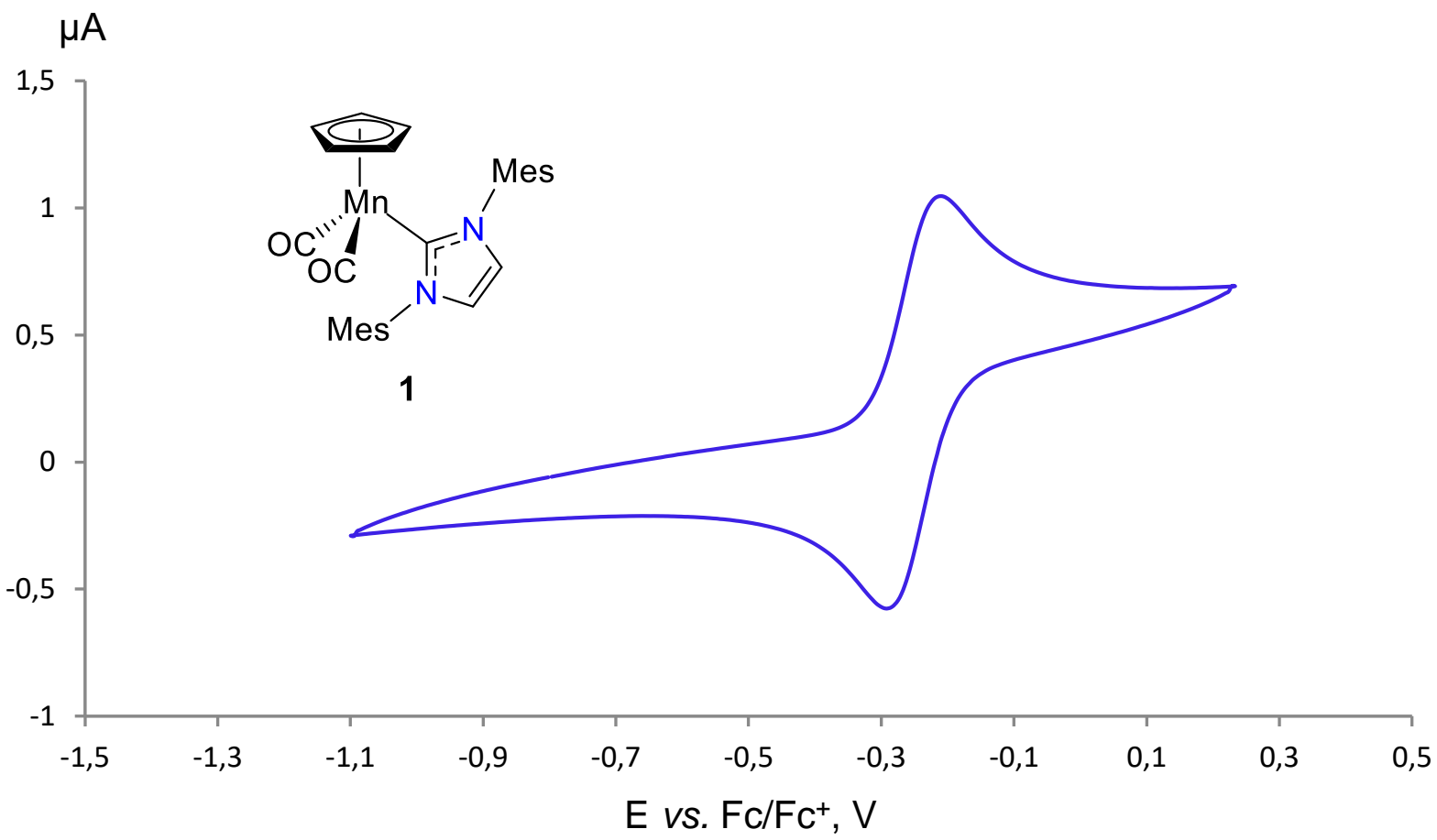

Figure S9. Cyclic voltammogram of complex $\mathrm{CpMn}(\mathrm{CO})_{2}(\mathrm{IMes})$ (1). Experimental conditions: $\mathrm{Pt}$ electrode, MeCN, $1 \mathrm{mM}$ sample concentration, $0.1 \mathrm{M} \mathrm{Bu}_{4} \mathrm{NPF}_{6}$ as supporting electrolyte, $200 \mathrm{mV} / \mathrm{s}$, potentials vs. Fc/Fc ${ }^{+}$couple.

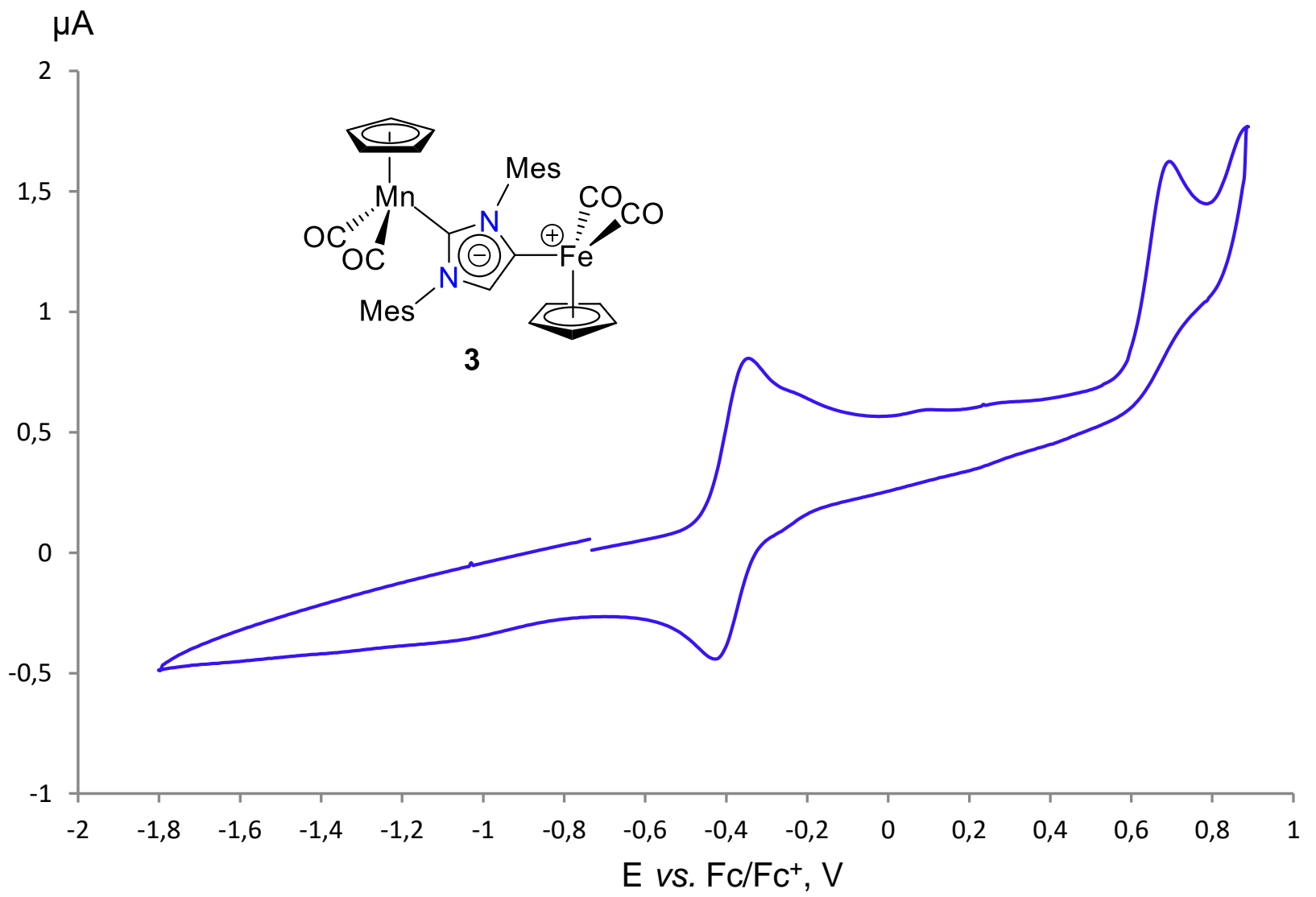

Figure S10. Cyclic voltammogram of complex $\mathrm{CpMn}(\mathrm{CO})_{2}\left(\mu\right.$-dMes) $\mathrm{Fe}(\mathrm{CO})_{2} \mathrm{Cp}$ (3). Experimental conditions: Pt electrode, MeCN, $1 \mathrm{mM}$ sample concentration, $0.1 \mathrm{M} \mathrm{Bu}_{4} \mathrm{NPF}_{6}$ as supporting electrolyte, $200 \mathrm{mV} / \mathrm{s}$, potentials vs. Fc/Fc ${ }^{+}$couple. 


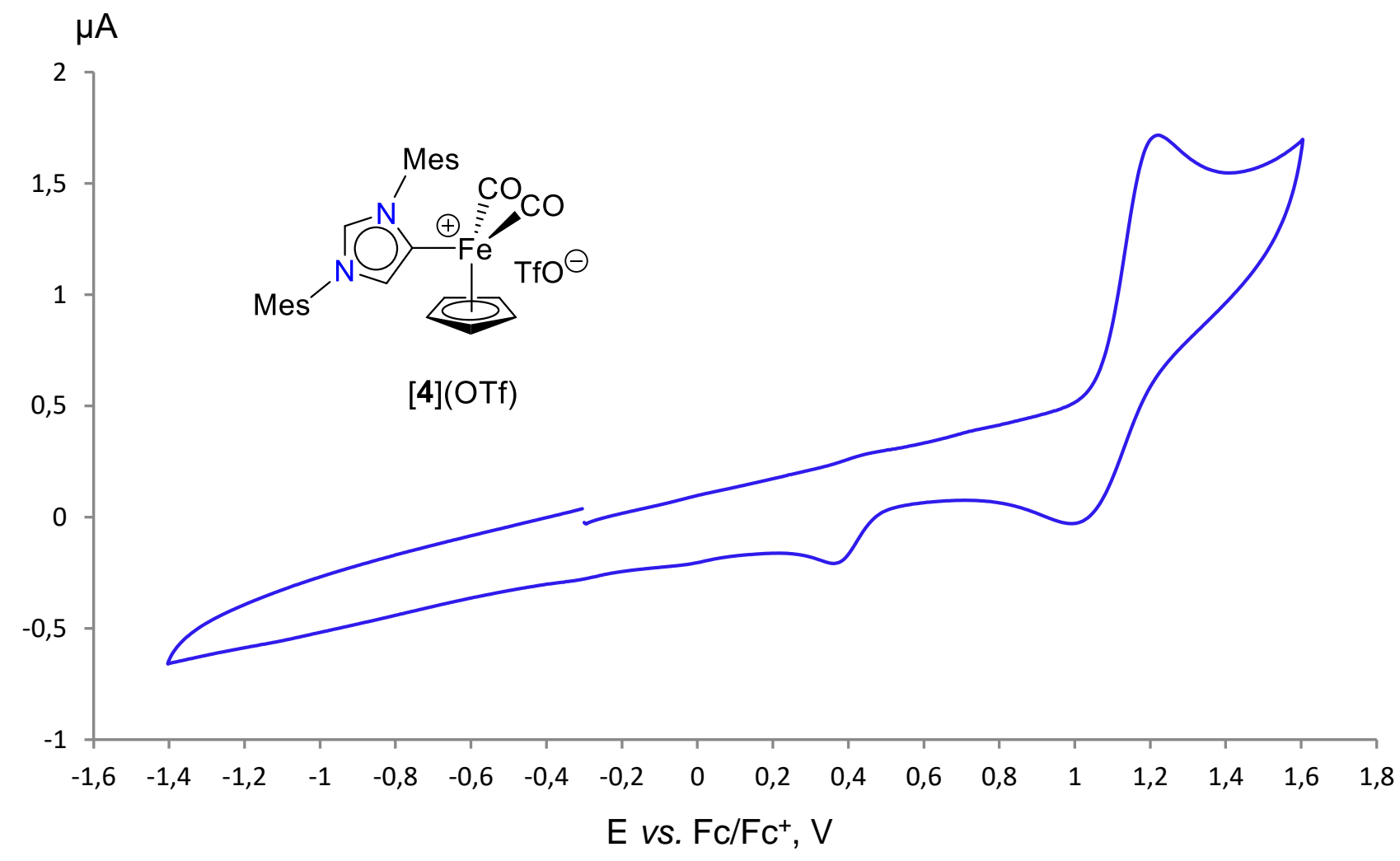

Figure S11. Cyclic voltammogram of complex $\left[\mathrm{CpFe}(\mathrm{CO})_{2}(\right.$ alMes $\left.)\right](\mathrm{OTf})$ ([4](OTf)). Experimental conditions: Pt electrode, MeCN, $1 \mathrm{mM}$ sample concentration, $0.1 \mathrm{M} \mathrm{Bu}_{4} \mathrm{NPF}_{6}$ as supporting electrolyte, $200 \mathrm{mV} / \mathrm{s}$, potentials vs. Fc/Fc couple.
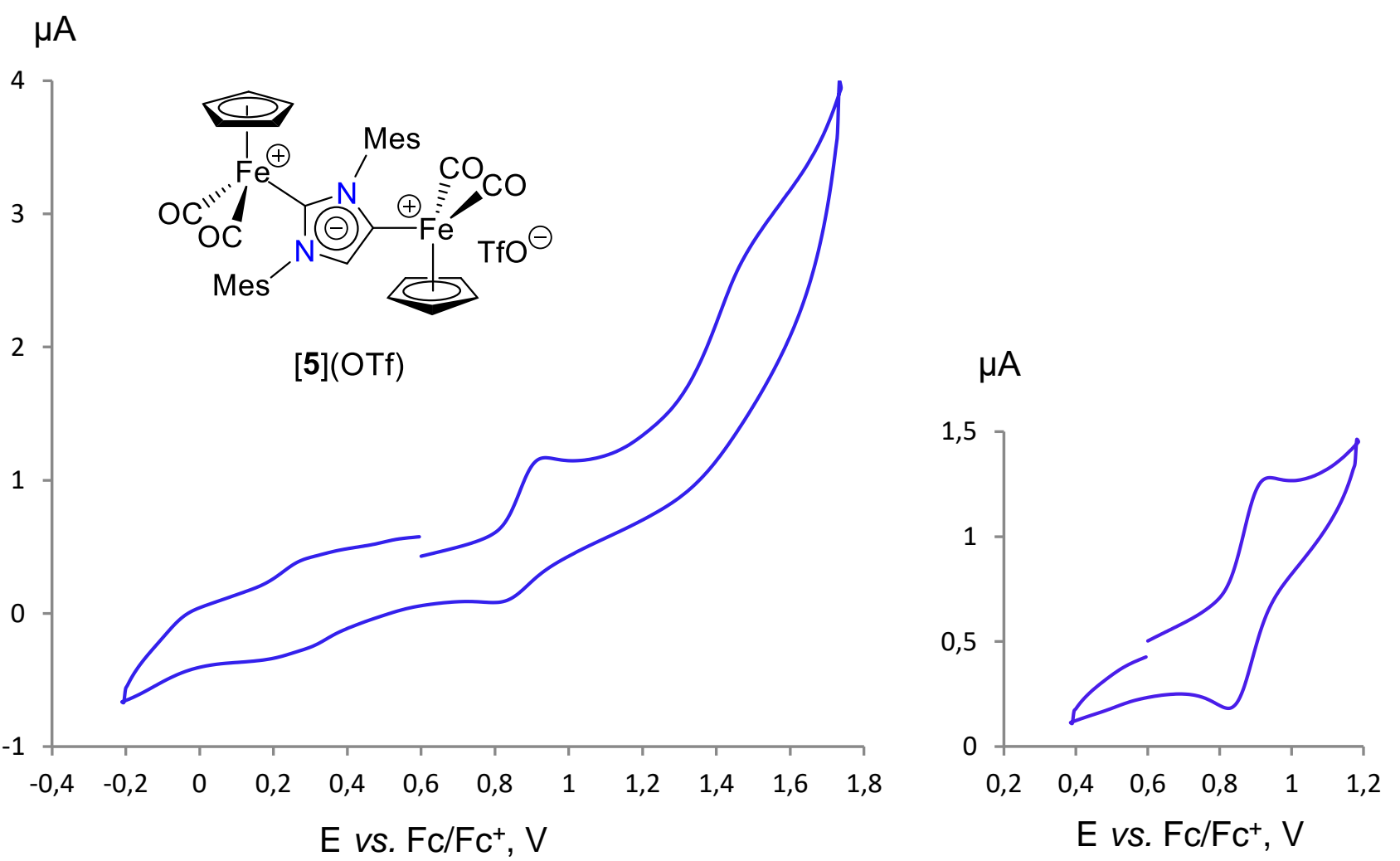

Figure S12. Cyclic voltammograms of complex $\left[\mathrm{CpFe}(\mathrm{CO})_{2}\left(\mu\right.\right.$-dlMes) $\left.\mathrm{Fe}(\mathrm{CO})_{2} \mathrm{Cp}\right](\mathrm{OTf})([5](\mathrm{OTf}))$. Experimental conditions: Pt electrode, MeCN, $1 \mathrm{mM}$ sample concentration, $0.1 \mathrm{M} \mathrm{Bu}_{4} \mathrm{NPF}_{6}$ as supporting electrolyte, $200 \mathrm{mV} / \mathrm{s}$, potentials vs. $\mathrm{Fc} / \mathrm{Fc}^{+}$couple. 


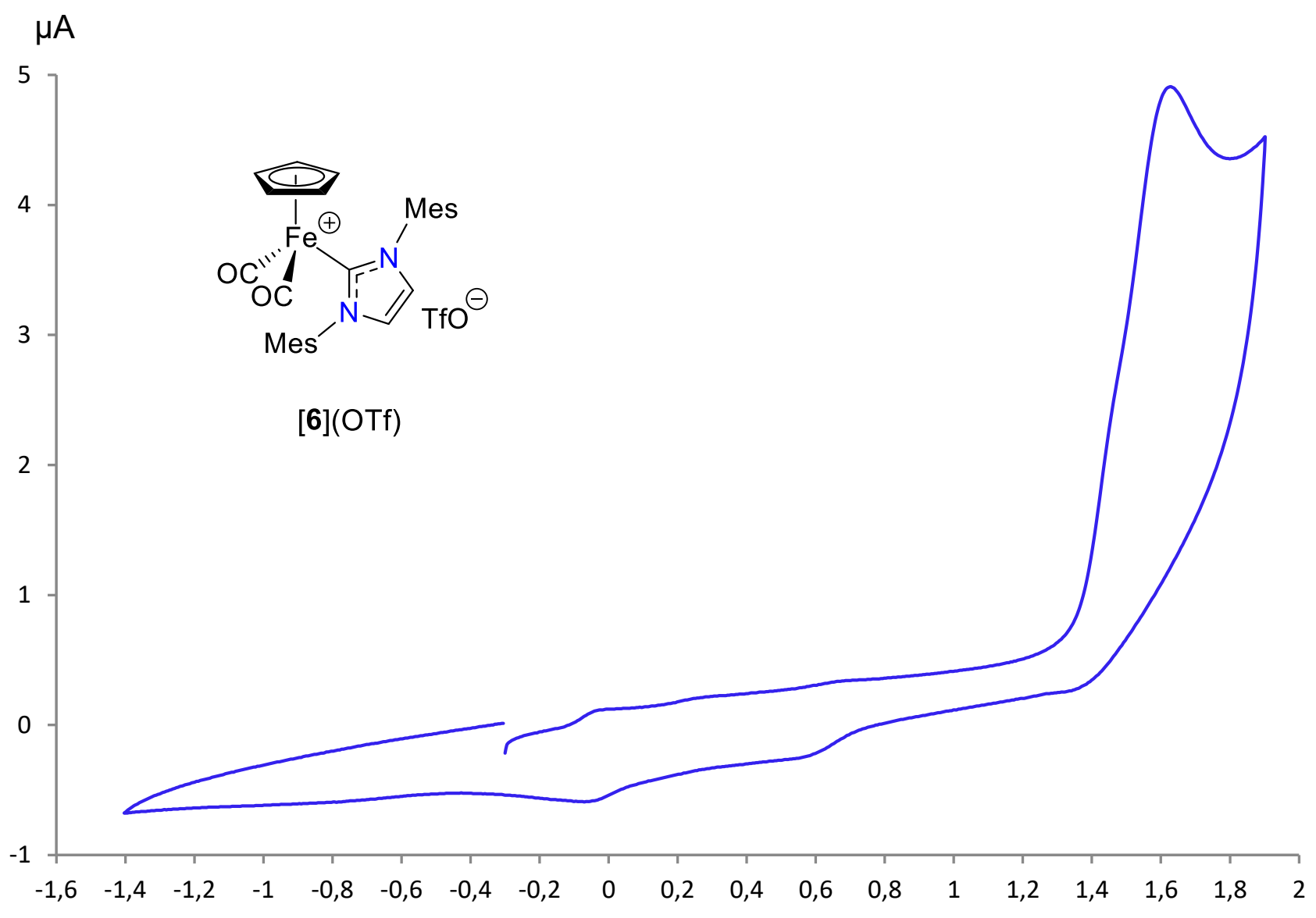

E vs. $\mathrm{Fc} / \mathrm{Fc}^{+}, \mathrm{V}$

Figure S13. Cyclic voltammogram of complex $\left[\mathrm{CpFe}(\mathrm{CO})_{2}(\mathrm{IMes})\right](\mathrm{OTf})([6](\mathrm{OTf}))$. Experimental conditions: Pt electrode, MeCN, $1 \mathrm{mM}$ sample concentration, $0.1 \mathrm{M} \mathrm{Bu}_{4} \mathrm{NPF}_{6}$ as supporting electrolyte, $200 \mathrm{mV} / \mathrm{s}$, potentials vs. $\mathrm{Fc} / \mathrm{Fc}^{+}$couple.2. Electrochemical studies of $\mathrm{Mn}(\mathrm{I})$ and $\mathrm{Fe}(\mathrm{II})$ $\mathrm{NHC}$ complexes 


\section{Computational results}

Table S1. Key structural data of calculated Mn(I) and Fe(II) NHC complexes (G09/BP86-D3/Def2-TZVP).

\begin{tabular}{|c|c|c|c|c|c|c|c|c|c|}
\hline Complex & $\mathrm{M} 1-\mathrm{NHC}$ & $\mathrm{C}_{i p s o \ldots . . . C 1-O 1}$ & $\mathrm{C}_{i p s o \ldots . . . C 2-\mathrm{O} 2}$ & $\mathrm{M} 1-\mathrm{C} 1-\mathrm{O} 1$ & $\mathrm{M} 1-\mathrm{C} 2-\mathrm{O} 2$ & $\mathrm{M} 2-\mathrm{aNHC}$ & $\mathrm{C}_{\text {ipso....C3-O3 }}$ & $\mathrm{M} 2-\mathrm{C} 3-\mathrm{O} 3$ & $\mathrm{M} 2-\mathrm{C} 4-\mathrm{O} 4$ \\
\hline $1 a$ & $1.979 \AA$ & $3.288 \AA$ & $3.084 \AA$ & $173.95^{\circ}$ & $172.43^{\circ}$ & - & - & - & - \\
\hline $1 b$ & $1.980 \AA$ & $3.938 \AA$ & $2.850 \AA$ & $177.28^{\circ}$ & $169.34^{\circ}$ & - & - & - & - \\
\hline $6 a^{+}$ & $1.976 \AA$ & $3.305 \AA$ & $2.965 \AA$ & $174.32^{\circ}$ & $171.00^{\circ}$ & - & - & - & - \\
\hline $6 b^{+}$ & $1.984 \AA$ & $3.820 \AA$ & $2.788 \AA$ & $176.60^{\circ}$ & $167.16^{\circ}$ & - & - & - & - \\
\hline $4^{+}$ & - & - & - & - & - & $1.967 \AA$ & $2.915 \AA$ & $170.84^{\circ}$ & $179.04^{\circ}$ \\
\hline $3 a$ & $1.988 \AA$ & $3.299 \AA$ & $3.046 \AA$ & $174.09 \AA$ & $172.07 \AA$ & $1.982 \AA$ & $2.932 \AA$ & $170.51^{\circ}$ & $179.67^{\circ}$ \\
\hline $3 b$ & $1.992 \AA$ & $3.840 \AA$ & $2.840 \AA$ & $176.79^{\circ}$ & $169.14^{\circ}$ & $1.987 \AA$ & $2.894 \AA$ & $169.34^{\circ}$ & $179.39^{\circ}$ \\
\hline $5 a^{+}$ & $1.985 \AA$ & $3.308 \AA$ & $2.934 \AA$ & $174.08^{\circ}$ & $170.55^{\circ}$ & $1.977 \AA$ & $2.920 \AA$ & $171.29^{\circ}$ & $178.99^{\circ}$ \\
\hline $5 b^{+}$ & $1.995 \AA$ & $3.749 \AA$ & $2.779 \AA$ & $176.53^{\circ}$ & $166.96^{\circ}$ & $1.981 \AA$ & $2.891 \AA$ & $170.52^{\circ}$ & $178.95^{\circ}$ \\
\hline
\end{tabular}

Table S2. Energy difference between the conformers $\mathrm{Mn}(\mathrm{I})$ and $\mathrm{Fe}(\mathrm{II}) \mathrm{NHC}$ complexes (G09/BP86D3/Def2-TZVP, horizontal isomers are taken as zero).

\begin{tabular}{|c|c|c|c|c|}
\hline Complex & $1 a / 1 b$ & $6 a^{+} / 6 b^{+}$ & $3 a / 3 b$ & $5 a^{+} / 5 b^{+}$ \\
\hline$\Delta \mathrm{E}, \mathrm{kcal} \mathrm{mol}^{-1}$ & -0.70 & -0.29 & 0.06 & -0.07 \\
\hline$\Delta \mathrm{E}_{\mathrm{ZPE}}, \mathrm{kcal} \mathrm{mol}^{-1}$ & -0.67 & -0.24 & 0.05 & -0.10 \\
\hline$\Delta \mathrm{E}_{\text {thermal }}, \mathrm{kcal} \mathrm{mol}^{-1}$ & -0.70 & -0.30 & -0.53 & -0.09 \\
\hline$\Delta \mathrm{H}^{298}, \mathrm{kcal} \mathrm{mol}^{-1}$ & -0.70 & -0.30 & -0.53 & -0.09 \\
\hline$\Delta \mathrm{G}^{298}, \mathrm{kcal} \mathrm{mol}^{-1}$ & -0.46 & 0.93 & 1.63 & 0.35 \\
\hline$\Delta \mathrm{S}^{298}, \mathrm{cal} \cdot \mathrm{mol}^{-1} \cdot \mathrm{K}^{-1}$ & -0.83 & -4.10 & -7.24 & -1.48 \\
\hline
\end{tabular}

Table S3. Characteristics of $B C P s$ for $\pi(C=C) \ldots \pi^{*}(C \equiv O)$ interligand interactions in optimized $M n(I)$ and $\mathrm{Fe}(\mathrm{II}) \mathrm{NHC}$ complexes (G09/BP86-D3/Def2-TZVP). ${ }^{a}$

\begin{tabular}{|c|c|c|c|c|c|c|c|c|c|c|c|c|c|}
\hline Complex & $1 \mathrm{a}$ & $1 b$ & $6 a^{+}$ & $6 b^{+}$ & $4^{+}$ & $\begin{array}{c}3 \mathbf{a} \\
\mathrm{NHC}\end{array}$ & $\begin{array}{c}\mathbf{3 a} \\
\mathrm{aNHC}\end{array}$ & $\begin{array}{c}3 \mathbf{b} \\
\mathrm{NHC}\end{array}$ & $\begin{array}{c}\mathbf{3 b} \\
\mathrm{aNHC}\end{array}$ & $\begin{array}{c}5 \mathbf{a}^{+} \\
\mathrm{NHC}\end{array}$ & $\begin{array}{c}\mathbf{5 a}^{+} \\
\mathrm{aNHC}\end{array}$ & $\begin{array}{c}5 b^{+} \\
\mathrm{NHC}\end{array}$ & $\begin{array}{c}\mathbf{5 b}^{+} \\
\mathrm{aNHC}\end{array}$ \\
\hline$\rho_{c}$ & - & 0.014 & 0.012 & 0.016 & 0.013 & - & 0.012 & 0.014 & 0.013 & 0.012 & 0.013 & 0.016 & 0.013 \\
\hline$\nabla^{2}\left(\rho_{c}\right)$ & - & 0.038 & 0.032 & 0.041 & 0.034 & - & 0.033 & 0.039 & 0.035 & 0.034 & 0.034 & 0.042 & 0.036 \\
\hline$\varepsilon_{\mathrm{c}}$ & - & 0.200 & 1.303 & 0.088 & 0.287 & - & 0.266 & 0.240 & 0.155 & 1.074 & 0.258 & 0.122 & 0.179 \\
\hline$V_{c}$ & - & -0.008 & -0.006 & -0.008 & -0.006 & - & -0.006 & -0.008 & -0.007 & -0.006 & -0.007 & -0.008 & -0.007 \\
\hline $\mathrm{G}_{\mathrm{c}}$ & - & 0.009 & 0.007 & 0.009 & 0.007 & - & 0.007 & 0.009 & 0.008 & 0.007 & 0.008 & 0.009 & 0.008 \\
\hline $\mathrm{K}_{\mathrm{c}}$ & - & -0.001 & -0.001 & -0.001 & -0.001 & - & -0.001 & -0.001 & -0.001 & -0.001 & -0.001 & -0.001 & -0.001 \\
\hline $\mathrm{L}_{c}$ & - & -0.010 & -0.008 & -0.010 & -0.008 & - & -0.008 & -0.010 & -0.009 & -0.008 & -0.009 & -0.010 & -0.009 \\
\hline DI & - & 0.043 & 0.035 & 0.054 & 0.038 & - & 0.037 & 0.043 & 0.041 & 0.037 & 0.036 & 0.053 & 0.039 \\
\hline $\mathrm{E}_{\mathrm{BCP}}$ & - & -2.4 & -1.9 & -2.6 & -2.0 & - & -2.0 & -2.4 & -2.1 & -2.0 & -2.1 & -2.6 & -2.2 \\
\hline
\end{tabular}

${ }^{a} \rho_{c}$ - electron density at BCP, a.u.; $\varepsilon$ - bond ellipticity; $\nabla^{2}\left(\rho_{c}\right)$ - Laplacian of Rho = trace of hessian of Rho; $V=$ virial field $=$ potential energy density = trace of stress tensor; $\mathrm{G}=$ Lagrangian form of kinetic energy density; $\mathrm{K}=$ Hamiltonian form of kinetic energy density $=\mathrm{L}=\mathrm{K}-\mathrm{G}=$ Lagrangian density $=(-1 / 4) \nabla^{2}\left(\rho_{\mathrm{C}}\right)$; $\mathrm{DI}=$ delocalization index = bond order; $\mathrm{E}_{\mathrm{BCP}}-$ energy of interaction, $\mathrm{kcal}^{\mathrm{mol}}{ }^{-1}$. 

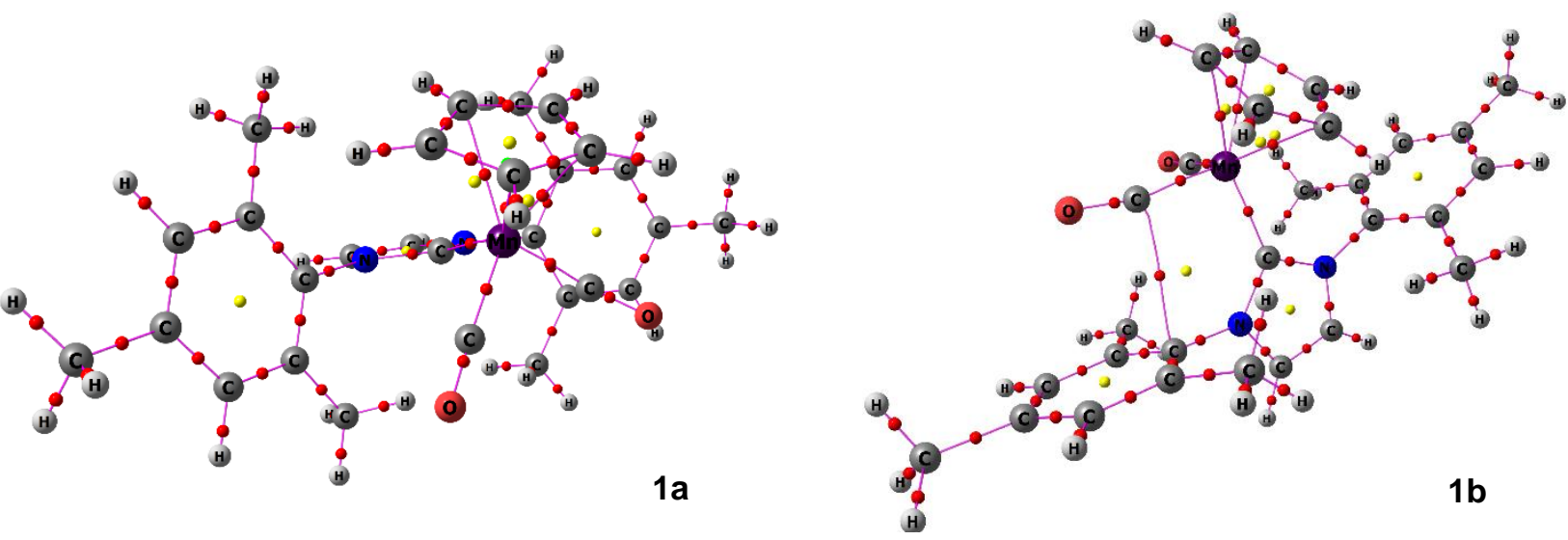

Figure S14. Molecular graphs for $\mathrm{Mn}(\mathrm{I}) \mathrm{IMes}$ complexes 1a (left) and 1b (right). Weak $\mathrm{C}-\mathrm{H} \cdots \mathrm{H}-\mathrm{C}$ and $\mathrm{C}-\mathrm{H} \cdots \mathrm{C} \equiv \mathrm{O}$ interactions with the energies below $1.5 \mathrm{kcal} \mathrm{mol}^{-1}$ are not shown for clarity. Pink - bond paths', red balls - bond $(3,-1)$ critical points, yellow balls - ring $(3,+1)$ critical points, green balls - cage $(3,+3)$ critical points.
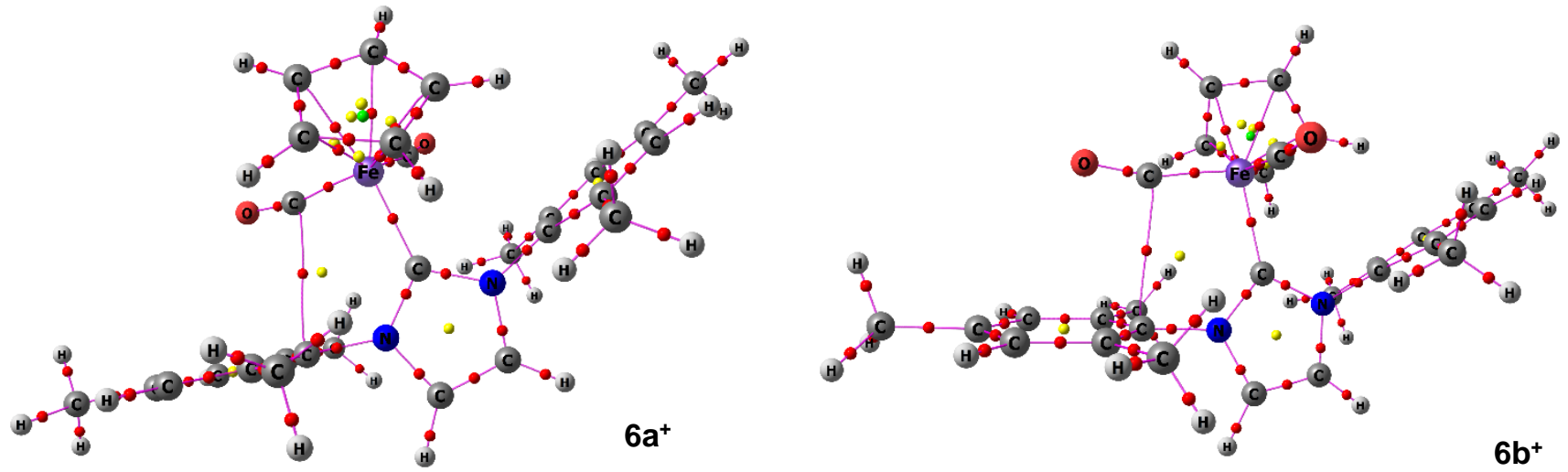

Figure S15. Molecular graphs for Fe(II) IMes complexes 6a+ (left) and 6a+ (right). Weak $\mathrm{C}-\mathrm{H} \cdots \mathrm{H}-\mathrm{C}$ and $\mathrm{C}-\mathrm{H} \cdots \mathrm{C} \equiv \mathrm{O}$ interactions with the energies below $1.5 \mathrm{kcal} \mathrm{mol}^{-1}$ are not shown for clarity. Pink - bond paths', red balls - bond $(3,-1)$ critical points, yellow balls - ring $(3,+1)$ critical points, green balls - cage $(3,+3)$ critical points.

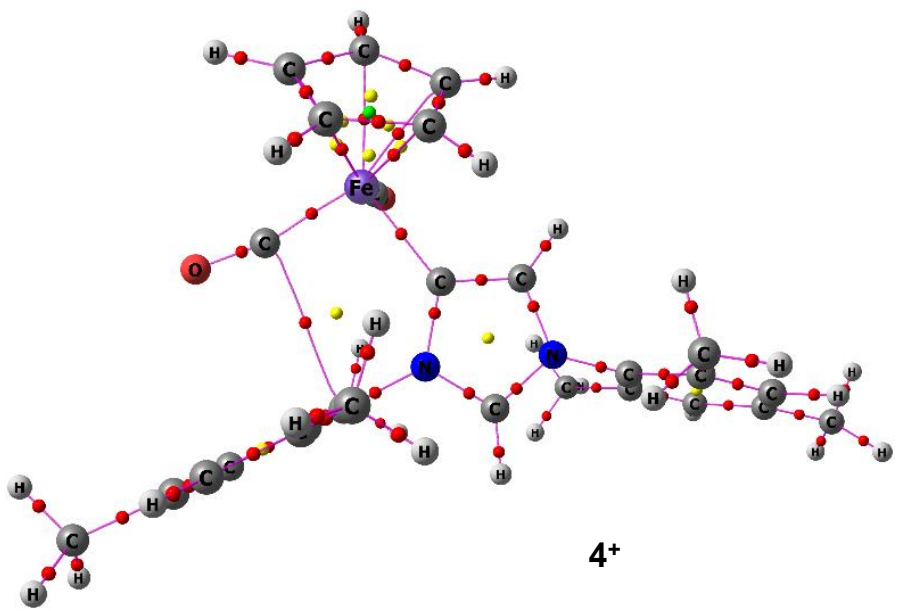

Figure S16. Molecular graphs for $\mathrm{Fe}(\mathrm{II})$ alMes complex $4^{+}$. Weak $\mathrm{C}-\mathrm{H} \cdots \mathrm{H}-\mathrm{C}$ and $\mathrm{C}-\mathrm{H} \cdots \mathrm{C} \equiv \mathrm{O}$ interactions with the energies below $1.5 \mathrm{kcal} \mathrm{mol}^{-1}$ are not shown for clarity. Pink - bond paths', red balls - bond $(3,-1)$ critical points, yellow balls - ring $(3,+1)$ critical points, green balls - cage $(3,+3)$ critical points. 

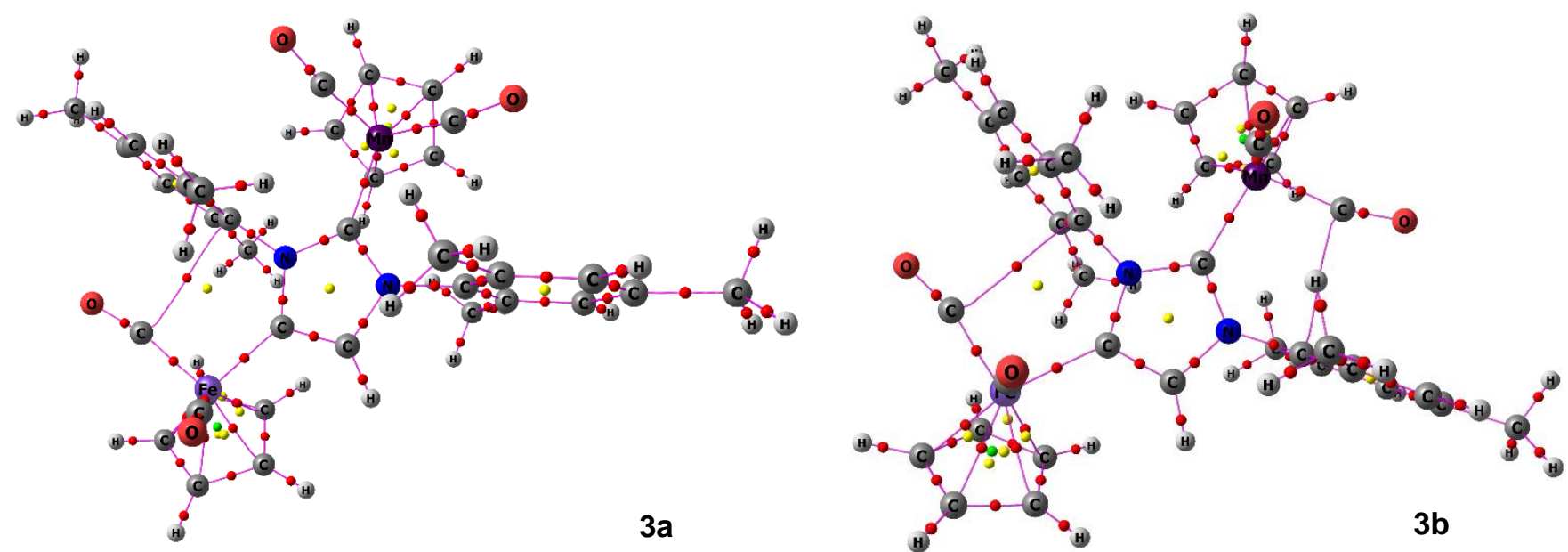

Figure S17. Molecular graphs for $\mathrm{Mn}(\mathrm{I}) / \mathrm{Fe}(\mathrm{II})$ dIMes complexes $\mathbf{3 a}$ (left) and $\mathbf{3 b}$ (right). Weak $\mathrm{C}-\mathrm{H} \cdots \mathrm{H}-\mathrm{C}$ and $\mathrm{C}-\mathrm{H} \cdots \mathrm{C} \equiv \mathrm{O}$ interactions with the energies below $1.5 \mathrm{kcal} \mathrm{mol}^{-1}$ are not shown for clarity. Pink - bond paths', red balls - bond $(3,-1)$ critical points, yellow balls - ring $(3,+1)$ critical points, green balls - cage $(3,+3)$ critical points.
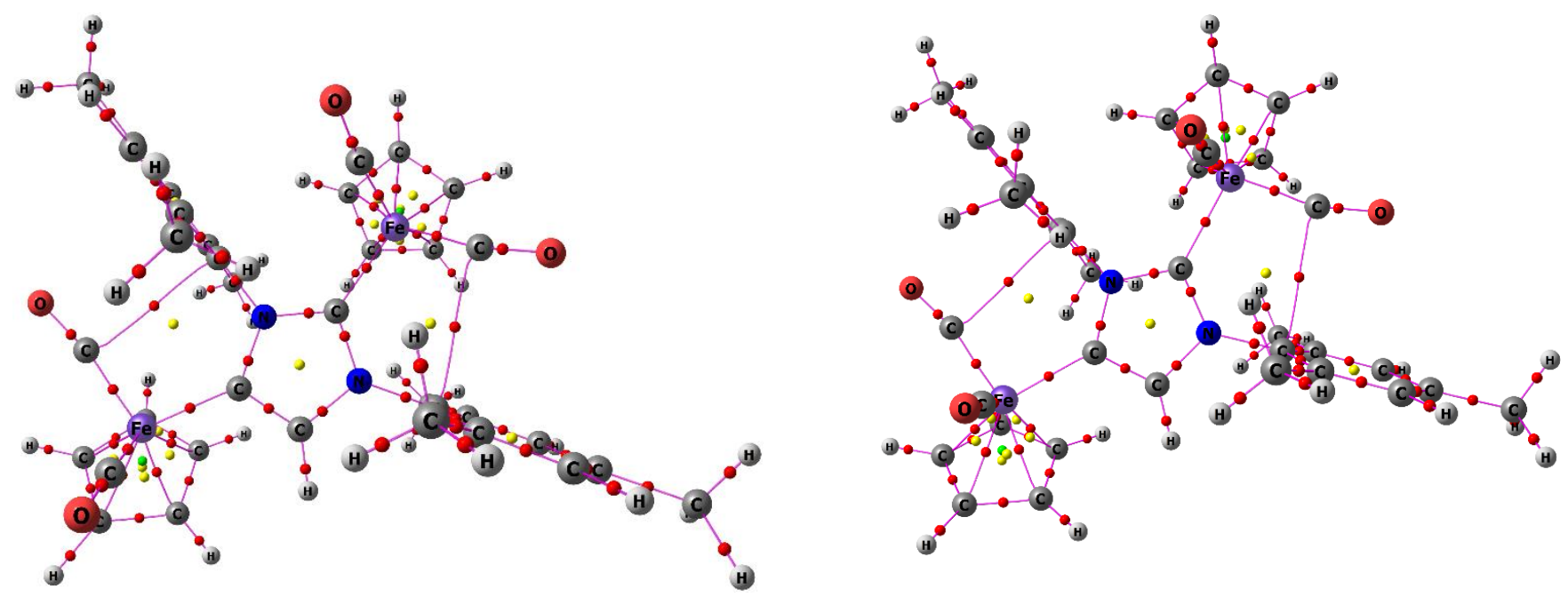

Figure S18. Molecular graphs for $\mathrm{Fe}(\mathrm{II}) \mathrm{dMes}$ complexes $\mathbf{5 \mathbf { a } ^ { + }}$ (left) and $\mathbf{5} \mathbf{b}^{+}$(right). Weak $\mathrm{C}-\mathrm{H} \cdots \mathrm{H}-\mathrm{C}$ and $\mathrm{C}-\mathrm{H} \cdots \mathrm{C} \equiv \mathrm{O}$ interactions with the energies below $1.5 \mathrm{kcal} \mathrm{mol}^{-1}$ are not shown for clarity. Pink - bond paths', red balls - bond $(3,-1)$ critical points, yellow balls - ring $(3,+1)$ critical points, green balls - cage $(3,+3)$ critical points. 
Table S4. Characteristics of BCPs for metal-carbene bonds in optimized $\mathrm{Mn}(\mathrm{I})$ and $\mathrm{Fe}(\mathrm{II}) \mathrm{NHC}$ complexes (G09/BP86-D3/Def2-TZVP). ${ }^{a}$

\begin{tabular}{|c|c|c|c|c|c|c|c|c|c|c|c|c|c|}
\hline Complex & $\begin{array}{c}\mathbf{1 a} \\
\mathrm{NHC}\end{array}$ & $\begin{array}{c}\text { 1b } \\
\mathrm{NHC}\end{array}$ & $\begin{array}{c}6 \mathbf{a}^{+} \\
\mathrm{NHC}\end{array}$ & $\begin{array}{c}6 \mathbf{b}^{+} \\
\mathrm{NHC}\end{array}$ & $\begin{array}{c}\mathbf{4}^{+} \\
\mathrm{aNHC}\end{array}$ & $\begin{array}{c}3 \mathbf{a} \\
\mathrm{NHC}\end{array}$ & $\begin{array}{c}3 \mathbf{a} \\
\mathrm{aNHC}\end{array}$ & $\begin{array}{c}\mathbf{3 b} \\
\mathrm{NHC}\end{array}$ & $\begin{array}{c}\mathbf{3 b} \\
\mathrm{aNHC}\end{array}$ & $\begin{array}{c}5 \mathbf{a}^{+} \\
\mathrm{NHC}\end{array}$ & $\begin{array}{c}\mathbf{5 a}^{+} \\
\mathrm{aNHC}\end{array}$ & $\begin{array}{c}5 \mathbf{b}^{+} \\
\mathrm{NHC}\end{array}$ & $\begin{array}{c}\mathbf{5 b}^{+} \\
\mathrm{aNHC}\end{array}$ \\
\hline$\rho_{c}$ & 0.108 & 0.107 & 0.111 & 0.109 & 0.114 & 0.107 & 0.112 & 0.104 & 0.111 & 0.110 & 0.112 & 0.106 & 0.111 \\
\hline$\nabla^{2}\left(\rho_{c}\right)$ & 0.301 & 0.314 & 0.238 & 0.243 & 0.215 & 0.289 & 0.168 & 0.303 & 0.168 & 0.222 & 0.196 & 0.227 & 0.195 \\
\hline$\varepsilon_{\mathrm{c}}$ & 0.036 & 0.086 & 0.022 & 0.056 & 0.065 & 0.037 & 0.057 & 0.081 & 0.081 & 0.024 & 0.066 & 0.056 & 0.082 \\
\hline $\mathrm{V}_{\mathrm{c}}$ & -0.146 & -0.147 & -0.144 & -0.141 & -0.145 & -0.142 & -0.133 & -0.141 & -0.131 & -0.139 & -0.138 & -0.135 & -0.137 \\
\hline $\mathrm{G}_{\mathrm{c}}$ & 0.111 & 0.113 & 0.102 & 0.101 & 0.099 & 0.107 & 0.088 & 0.108 & 0.087 & 0.097 & 0.094 & 0.096 & 0.093 \\
\hline $\mathrm{K}_{\mathrm{c}}$ & 0.036 & 0.034 & 0.042 & 0.040 & 0.045 & 0.035 & 0.046 & 0.033 & 0.045 & 0.042 & 0.045 & 0.039 & 0.044 \\
\hline$L_{c}$ & -0.075 & -0.079 & -0.060 & -0.061 & -0.054 & -0.072 & -0.042 & -0.076 & -0.042 & -0.055 & -0.049 & -0.057 & -0.049 \\
\hline DI & 0.800 & 0.813 & 0.782 & 0.779 & 0.799 & 0.787 & 0.791 & 0.795 & 0.787 & 0.776 & 0.792 & 0.769 & 0.789 \\
\hline
\end{tabular}

${ }^{a} \rho_{c}$ - electron density at BCP, a.u.; $\varepsilon$ - bond ellipticity; $\nabla^{2}\left(\rho_{c}\right)$ - Laplacian of Rho = trace of hessian of Rho; $V=$ virial field $=$ potential energy density = trace of stress tensor; $\mathrm{G}=$ Lagrangian form of kinetic energy density; $\mathrm{K}=$ Hamiltonian form of kinetic energy density $=\mathrm{L}=\mathrm{K}-$ $\mathrm{G}=$ Lagrangian density $=(-1 / 4) \nabla^{2}\left(\rho_{\mathrm{c}}\right) ; \mathrm{DI}=$ delocalization index = bond order.

Table S5. Selected AIM charges for optimized Mn(I) and Fe(II) NHC complexes.

\begin{tabular}{lccccccccc}
\hline Complex & $\mathbf{1 a}$ & $\mathbf{1 b}$ & $\mathbf{6 \mathbf { a } ^ { + }}$ & $\mathbf{6 \mathbf { b } ^ { + }}$ & $\mathbf{\mathbf { 4 } ^ { + }}$ & $\mathbf{3 a}$ & $\mathbf{3 b}$ & $\mathbf{5 \mathbf { a } ^ { + }}$ & $\mathbf{5 \mathbf { b } ^ { + }}$ \\
\hline $\mathrm{M}-\mathrm{NHC}$ & +0.948 & +0.946 & +0.845 & +0.843 & - & +0.951 & +0.947 & +0.845 & +0.844 \\
$\mathrm{M}-\mathrm{aNHC}$ & - & - & - & - & +0.840 & +0.838 & +0.840 & +0.840 & +0.841 \\
$\mathrm{CpM}(\mathrm{CO})_{2}-\mathrm{NHC}^{a}$ & -0.142 & -0.134 & +0.561 & +0.550 & - & -0.157 & -0.151 & +0.523 & +0.514 \\
$\mathrm{CpM}(\mathrm{CO})_{2}-\mathrm{aNHC}^{a}$ & - & - & - & - & +0.502 & +0.345 & +0.344 & +0.456 & +0.457 \\
$\mathrm{NHC}$ & -0.799 & -0.806 & -0.647 & -0.649 & -0.616 & -1.115 & -1.123 & -1.027 & -1.031 \\
$\mathrm{C} 2$ & +0.601 & +0.590 & +0.660 & +0.653 & +0.872 & +0.596 & +0.587 & +0.649 & +0.642 \\
$\mathrm{C} 4$ & $+0.286^{c}$ & $+0.286^{c}$ & $+0.308^{c}$ & $+0.309^{c}$ & +0.178 & +0.142 & +0.141 & +0.154 & +0.156 \\
\hline
\end{tabular}

${ }^{a}$ total AIM charge of the $\mathrm{Cp}(\mathrm{CO})_{2} \mathrm{M}$ moiety

${ }^{b}$ total AIM charge of the heterocyclic moiety without aryl groups $\left(\mathrm{C}_{3} \mathrm{~N}_{2} \mathrm{H}_{2}\right.$ and $\mathrm{C}_{3} \mathrm{~N}_{2} \mathrm{H}_{1}$ for mono- and ditopic carbenes, respectively)

${ }^{c}$ for normal carbene complexes average charge of $\mathrm{C} 4$ and $\mathrm{C} 5$ is provided due to slight asymmetry

Table S6. Selected NBO6 charges for optimized $\mathrm{Mn}(\mathrm{I})$ and $\mathrm{Fe}(\mathrm{II}) \mathrm{NHC}$ complexes (G09/BP86-D3/Def2-TZVP).

\begin{tabular}{lccccccccc}
\hline Complex & $\mathbf{1 a}$ & $\mathbf{1 b}$ & $\mathbf{6 \mathbf { a } ^ { + }}$ & $\mathbf{6 \mathbf { b } ^ { + }}$ & $\mathbf{4}^{+}$ & $\mathbf{3 a}$ & $\mathbf{3 b}$ & $\mathbf{5 \mathbf { a } ^ { + }}$ & $\mathbf{5} \mathbf{b}^{+}$ \\
\hline $\mathrm{M}-\mathrm{NHC}$ & -0.576 & -0.565 & -0.241 & -0.230 & - & -0.570 & -0.557 & -0.238 & -0.226 \\
$\mathrm{M}-\mathrm{aNHC}$ & - & - & - & - & -0.240 & -0.239 & -0.235 & -0.239 & -0.236 \\
$\mathrm{CpM}(\mathrm{CO})_{2}-\mathrm{NHC}^{a}$ & -0.386 & -0.373 & +0.441 & +0.446 & - & -0.392 & -0.380 & +0.416 & +0.412 \\
$\mathrm{CpM}(\mathrm{CO})_{2}-\mathrm{aNHC}^{a}$ & - & - & - & - & +0.394 & +0.271 & +0.272 & +0.362 & +0.364 \\
$\mathrm{NHC} C^{b}$ & -0.099 & -0.111 & -0.007 & -0.015 & 0.019 & -0.358 & -0.375 & -0.322 & -0.334 \\
$\mathrm{C} 2$ & +0.407 & +0.393 & +0.377 & +0.365 & +0.191 & +0.394 & +0.381 & +0.361 & +0.346 \\
$\mathrm{C} 4$ & $-0.101^{c}$ & $-0.100^{c}$ & $-0.079^{c}$ & $-0.077^{c}$ & +0.097 & +0.079 & +0.075 & +0.081 & +0.082 \\
\hline
\end{tabular}

${ }^{a}$ total NBO charge of the $\mathrm{Cp}(\mathrm{CO})_{2} \mathrm{M}$ moiety

${ }^{b}$ total NBO charge of the heterocyclic moiety without aryl groups $\left(\mathrm{C}_{3} \mathrm{~N}_{2} \mathrm{H}_{2}\right.$ and $\mathrm{C}_{3} \mathrm{~N}_{2} \mathrm{H}_{1}$ for mono- and ditopic carbenes, respectively)

${ }^{c}$ for normal carbene complexes average charge of $\mathrm{C} 4$ and $\mathrm{C} 5$ is provided due to slight asymmetry 
Table S7. Calculated vco bands for optimized Mn(I) and Fe(II) NHC complexes.

\begin{tabular}{|c|c|c|c|c|c|c|c|c|c|c|c|c|c|}
\hline Complex & $1 \mathrm{a}$ & $1 b$ & $6 a^{+}$ & $6 b^{+}$ & $4^{+}$ & $\begin{array}{c}3 \mathbf{a} \\
\mathrm{M}-\mathrm{NHC}\end{array}$ & $\begin{array}{c}3 a \\
M-a N H C\end{array}$ & $\begin{array}{c}3 \mathbf{b} \\
\mathrm{M}-\mathrm{NHC}\end{array}$ & $\begin{array}{c}3 \mathbf{b} \\
\mathrm{M}-\mathrm{aNHC}\end{array}$ & $\begin{array}{c}5 \mathbf{a}^{+} \\
\mathrm{M}-\mathrm{NHC}\end{array}$ & $\begin{array}{c}5 \mathbf{a}^{+} \\
\mathrm{M}-\mathrm{aNHC}\end{array}$ & $\begin{array}{c}5 b^{+} \\
M-N H C\end{array}$ & $\begin{array}{c}\mathbf{5 b}^{+} \\
\mathrm{M}-\mathrm{aNHC}\end{array}$ \\
\hline \multicolumn{14}{|l|}{ G09a } \\
\hline$v_{1}, \mathrm{~cm}^{-1}$ & 1875 & 1872 & 1999 & 1993 & 1993 & 1873 & 1975 & 1869 & 1974 & 1994 & 1986 & 1987 & 1984 \\
\hline $\mathrm{A}_{1}, \mathrm{~km} \mathrm{~mol}^{-1}$ & 831 & 669 & 564 & 476 & 524 & 740 & 523 & 615 & 504 & 639 & 364 & 408 & 482 \\
\hline$v_{2}, \mathrm{~cm}^{-1}$ & 1923 & 1922 & 2038 & 2031 & 2033 & 1919 & 2017 & 1918 & 2016 & 2034 & 2026 & 2029 & 2023 \\
\hline $\mathrm{A}_{2}, \mathrm{~km} \mathrm{~mol}^{-1}$ & 558 & 627 & 390 & 407 & 438 & 621 & 492 & 652 & 449 & 618 & 243 & 609 & 265 \\
\hline \multicolumn{14}{|l|}{$\Delta \mathrm{G} 09,{ }^{b} \mathrm{~cm}^{-1}$} \\
\hline$v_{1}$ & -39 & -36 & 7 & 14 & 3 & -46 & 5 & -42 & 6 & 4 & 5 & 11 & 7 \\
\hline$v_{2}$ & -15 & -14 & 13 & 19 & 11 & -19 & 13 & -17 & 14 & 10 & 12 & 15 & 14 \\
\hline \multicolumn{14}{|l|}{$\mathrm{ADF}^{c}$} \\
\hline$v_{1}, \mathrm{~cm}^{-1}$ & 1854 & 1851 & 1979 & 1972 & 1973 & 1850 & 1954 & 1848 & 1953 & 1973 & 1964 & 1963 & 1966 \\
\hline $\mathrm{A}_{1}, \mathrm{~km} \mathrm{~mol}^{-1}$ & 860 & 698 & 582 & 493 & 556 & 773 & 564 & 641 & 528 & 658 & 396 & 516 & 388 \\
\hline$v_{2}, \mathrm{~cm}^{-1}$ & 1903 & 1903 & 2018 & 2011 & 2013 & 1898 & 1996 & 1898 & 1996 & 2015 & 2005 & 2002 & 2009 \\
\hline $\mathrm{A}_{2}, \mathrm{~km} \mathrm{~mol}^{-1}$ & 591 & 646 & 417 & 422 & 457 & 659 & 515 & 673 & 472 & 649 & 264 & 315 & 599 \\
\hline \multicolumn{14}{|l|}{$\Delta \mathrm{ADF},{ }^{b} \mathrm{~cm}^{-1}$} \\
\hline$v_{1}$ & -18 & -15 & 28 & 34 & 23 & -23 & 26 & -21 & 27 & 25 & 27 & 35 & 25 \\
\hline$v_{2}$ & 6 & 6 & 32 & 39 & 31 & 2 & 34 & 3 & 34 & 29 & 33 & 42 & 29 \\
\hline
\end{tabular}

${ }^{a}$ G09/BP86-D3/Def2-TZVP level.

${ }^{b} \Delta=v_{C O}{ }^{\text {exp }}-v_{\text {CO }}$ calc

${ }^{c}$ ADF/BP86-D3/TZP level.

Table S8. ETS-NOCV analysis data for metal-carbene bonding in optimized $\mathrm{Mn}(\mathrm{I})$ and $\mathrm{Fe}(\mathrm{II}) \mathrm{NHC}$ complexes bearing IMes, alMes and dIMes ligands (ADF/BP86-D3/TZP, energies are given in $\mathrm{kcal} \mathrm{mol}^{-1}$ ).

\begin{tabular}{|c|c|c|c|c|c|c|c|c|c|c|c|c|c|}
\hline Complex & $1 a$ & $1 b$ & $6 a^{+}$ & $6 b^{+}$ & $4^{+}$ & $\begin{array}{c}3 \mathbf{a} \\
\mathrm{M}-\mathrm{NHC}\end{array}$ & $\begin{array}{c}3 \mathbf{a} \\
\mathrm{M}-\mathrm{aNHC}\end{array}$ & $\begin{array}{c}3 b \\
M-N H C\end{array}$ & $\begin{array}{c}3 \mathbf{b} \\
\mathrm{M}-\mathrm{aNHC}\end{array}$ & $\begin{array}{c}5 \mathbf{a}^{+} \\
\mathrm{M}-\mathrm{NHC}\end{array}$ & $\begin{array}{c}5 \mathbf{a}^{+} \\
\mathrm{M}-\mathrm{aNHC}\end{array}$ & $\begin{array}{c}5 \mathbf{b}^{+} \\
\mathrm{M}-\mathrm{NHC}\end{array}$ & $\begin{array}{c}5 b^{+} \\
M-a N H C\end{array}$ \\
\hline EPauli & 141.5 & 146.2 & 144.1 & 145.1 & 155.3 & 139.1 & 172.7 & 144.7 & 173.0 & 141.7 & 150.5 & 145.5 & 149.9 \\
\hline$E_{\text {elst }}$ & -126.4 & -129.5 & -145.3 & -144.9 & -160.9 & -123.5 & -229.3 & -127.5 & -228.7 & -145.6 & -154.8 & -146.5 & -153.2 \\
\hline$E_{\text {disp }}$ & -22.9 & -23.6 & -23.8 & -24.1 & -16.0 & -23.7 & -17.4 & -24.3 & -17.4 & -24.7 & -17.2 & -24.9 & -17.4 \\
\hline$E_{\text {int }}$ & -73.6 & -75.3 & -112.7 & -113.1 & -117.5 & -73.8 & -188.9 & -75.6 & -188.6 & -120.1 & -125.2 & -120.3 & -124.6 \\
\hline Eorb & -65.8 & -68.5 & -87.8 & -89.2 & -95.9 & -65.8 & -114.9 & -68.5 & -115.4 & -91.6 & -103.6 & -94.4 & -103.8 \\
\hline$E_{\text {steric }}=E_{\text {Pauli }}+E_{\text {dst }}$ & 15.1 & 16.7 & -1.2 & 0.2 & -5.6 & 15.6 & -56.6 & 17.2 & -55.7 & -3.9 & -4.3 & -1.0 & -3.3 \\
\hline$E_{\text {orb }} / E_{\text {int }}$ & 0.89 & 0.91 & 0.78 & 0.79 & 0.82 & 0.89 & 0.61 & 0.91 & 0.61 & 0.76 & 0.83 & 0.78 & 0.83 \\
\hline $\mathrm{E}_{\text {elstat }} / \mathrm{E}_{\text {int }}$ & 1.72 & 1.72 & 1.29 & 1.28 & 1.37 & 1.67 & 1.21 & 1.69 & 1.21 & 1.21 & 1.24 & 1.22 & 1.23 \\
\hline $\mathrm{E}_{\sigma}(\mathrm{C}: \rightarrow \mathrm{M})$ & -38.0 & -38.8 & -54.3 & -53.9 & -64.8 & -38.6 & -78.2 & -39.4 & -77.9 & -57.3 & -71.5 & -57.5 & -71.3 \\
\hline$E_{\sigma}(C: \rightarrow M) / E_{o r b}$ & 0.58 & 0.57 & 0.62 & 0.60 & 0.68 & 0.59 & 0.68 & 0.58 & 0.68 & 0.63 & 0.69 & 0.61 & 0.69 \\
\hline $\mathrm{E}_{\Pi \perp}(\mathrm{M} \rightarrow \mathrm{C}:)$ & -12.5 & -13.9 & -9.6 & -9.8 & -9.1 & -11.2 & -8.7 & -12.9 & -8.9 & -9.2 & -8.7 & -9.9 & -9.0 \\
\hline $\mathrm{E}_{\pi \perp}(\mathrm{M} \rightarrow \mathrm{C}:) / \mathrm{E}_{\text {orb }}$ & 0.19 & 0.20 & 0.11 & 0.11 & 0.09 & 0.17 & 0.08 & 0.19 & 0.08 & 0.10 & 0.08 & 0.10 & 0.09 \\
\hline$E_{\pi \|}(M \rightarrow C:)$ & -5.7 & -5.4 & -3.8 & $\begin{array}{l}-4.1 \\
-2.2^{a}\end{array}$ & -4.4 & -5.7 & $\begin{array}{l}-2.5 \\
-2.8 \\
-4.2^{a}\end{array}$ & -5.5 & $\begin{array}{l}-3.5 \\
-4.0^{a}\end{array}$ & -4.1 & -3.2 & $\begin{array}{l}-4.1 \\
-2.4^{a}\end{array}$ & -3.4 \\
\hline $\begin{array}{l}E \pi(C=C) \rightarrow \\
\pi^{*}(C \equiv O)\end{array}$ & - & -1.5 & -3.3 & -4.3 & -3.7 & - & -4.1 & -1.5 & -4.6 & -3.2 & -5.1 & -4.2 & -5.0 \\
\hline
\end{tabular}

${ }^{a}$ additional NOCV channels responsible for metal-NHC $\pi$-backbonding, which can not be reliably attributed to the orthogonal $\Pi \perp$ or parallel $\pi \|$ components 
Comparison of metal-carbon bonding between model $\sigma$-complexes $\mathrm{Cp}(\mathrm{CO})_{2} \mathrm{Fe}-\mathrm{R}$ (7-9) and $\mathrm{Fe}$ (II) carbene complexes. Since transition metal NHDC complexes can be drawn as two resonance forms (Scheme S1 (left), both representations are equally used in literature), in which ditopic ligand formally belongs to anionic $L_{2}$ and neutral $L X$-type system, we decided to compare the nature of Fe-carbene bonds in dMes complexes 3 and $\mathbf{5}^{+}$with classic iron-carbon $\sigma$-bond in model complexes 7-9 (Scheme S1, right). The optimized geometries of the later species calculated at G09/BP86-D3/Def2-TZVP level are presented in Figure S19. The Fe-C bond distances in both $\sigma$-imidazolyl derivatives (7: $1.977 \AA$; 8: $1.984 \AA$ ) are very close to those of $\mathrm{Fe}-\mathrm{NHC}$ and $\mathrm{Fe}-\mathrm{aNHC}$ bonds in complexes 3 and $[4-6]^{+}$, whereas this bond is rather longer in case of $\sigma$-methyl complex $9(2.068 \AA)$. The AlM analysis of metal-carbon bonding (Table S9) in 7-9 revealed virtually the same metal-carbon bond orders as found in the corresponding $\mathrm{NHC}$ derivatives. However, the values of Laplacian $\nabla^{2}\left(\rho_{c}\right)$ for $\mathrm{Fe}-\mathrm{C}$ bonds gradually decrease in the row Fe-NHC $\rightarrow \mathrm{Fe}-a \mathrm{NHC} \rightarrow \mathrm{Fe}$-heteroaryl $\rightarrow \mathrm{Fe}$-alkyl (Table S9) being consistent with the increase of ionic bond character from left to right.

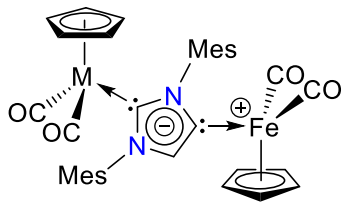

anionic $L_{2}$-type

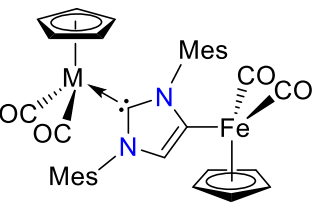

neutral LX-type<smiles>Cn1ccnc1P(=O)([O-])O[Na]</smiles>

7<smiles></smiles>

8<smiles>O=[Te]1([O-])([Mg])C=C1</smiles>

9 $\mathrm{M}=\mathrm{Mn}(3), \mathrm{Fe}^{+}\left(5^{+}\right)$

Scheme S1. Two possible resonance forms for transition metal NHDC complexes (left) and model $\sigma$ complexes $\mathrm{Cp}(\mathrm{CO})_{2} \mathrm{Fe}-\mathrm{R}(7-9$, right) used for the comparative theoretical investigation of $\mathrm{Fe}-\mathrm{C} 4$ bonding.
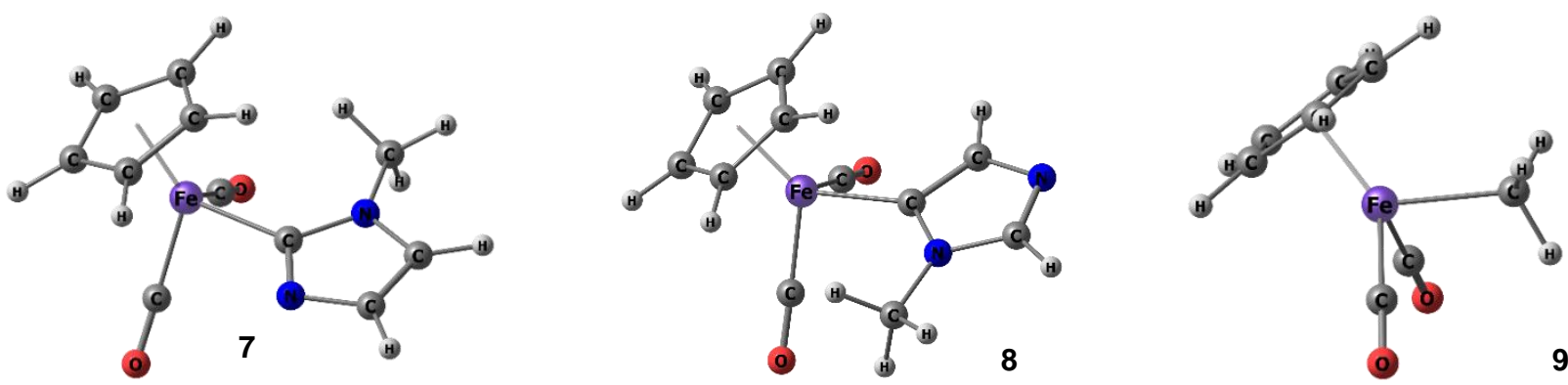

Figure S19. DFT optimized geometries for Fe(II) complexes 7 (left), 8 (center) and $\mathbf{9}$ (right) calculated at G09/BP86-D3/def2-TZVP level.

Table S9. Comparison of the selected characteristics of BCPs for $\mathrm{Fe}-\mathrm{C}$ bonds in optimized $\mathrm{Fe}$ (II) $\sigma$-complexes 7-9 with those of Fe-NHC bonds in IMes, alMes and dlMes complexes (G09/BP86D3/Def2-TZVP). ${ }^{a}$

\begin{tabular}{|c|c|c|c|c|c|c|c|c|c|c|c|c|}
\hline Complex & $\begin{array}{c}6 \mathbf{a}^{+} \\
\mathrm{NHC}\end{array}$ & $\begin{array}{c}6 \mathbf{b}^{+} \\
\mathrm{NHC}\end{array}$ & $\begin{array}{c}\mathbf{4}^{+} \\
\mathrm{aNHC}\end{array}$ & $\begin{array}{c}\mathbf{3 a} \\
\mathrm{aNHC}\end{array}$ & $\begin{array}{c}3 \mathbf{b} \\
\mathrm{aNHC}\end{array}$ & $\begin{array}{c}5 \mathbf{a}^{+} \\
\mathrm{NHC}\end{array}$ & $\begin{array}{c}\mathbf{5 a}^{+} \\
\mathrm{aNHC}\end{array}$ & $\begin{array}{c}5 \mathbf{b}^{+} \\
\mathrm{NHC}\end{array}$ & $\begin{array}{c}\mathbf{5 b}^{+} \\
\mathrm{aNHC}\end{array}$ & 7 & 8 & 9 \\
\hline$\rho_{c}$ & 0.111 & 0.109 & 0.114 & 0.112 & 0.111 & 0.110 & 0.112 & 0.106 & 0.111 & 0.115 & 0.112 & 0.095 \\
\hline$\nabla^{2}\left(\rho_{c}\right)$ & 0.238 & 0.243 & 0.215 & 0.168 & 0.168 & 0.222 & 0.196 & 0.227 & 0.195 & 0.152 & 0.146 & 0.091 \\
\hline$\varepsilon_{\mathrm{c}}$ & 0.022 & 0.056 & 0.065 & 0.057 & 0.081 & 0.024 & 0.066 & 0.056 & 0.082 & 0.042 & 0.014 & 0.027 \\
\hline $\mathrm{V}_{\mathrm{c}}$ & -0.144 & -0.141 & -0.145 & -0.133 & -0.131 & -0.139 & -0.138 & -0.135 & -0.137 & -0.135 & -0.130 & -0.092 \\
\hline $\mathrm{G}_{c}$ & 0.102 & 0.101 & 0.099 & 0.088 & 0.087 & 0.097 & 0.094 & 0.096 & 0.093 & 0.086 & 0.083 & 0.057 \\
\hline $\mathrm{K}_{\mathrm{c}}$ & 0.042 & 0.040 & 0.045 & 0.046 & 0.045 & 0.042 & 0.045 & 0.039 & 0.044 & 0.048 & 0.047 & 0.035 \\
\hline $\mathrm{L}_{c}$ & -0.060 & -0.061 & -0.054 & -0.042 & -0.042 & -0.055 & -0.049 & -0.057 & -0.049 & -0.038 & -0.036 & -0.023 \\
\hline DI & 0.782 & 0.779 & 0.799 & 0.791 & 0.787 & 0.776 & 0.792 & 0.769 & 0.789 & 0.781 & 0.795 & 0.793 \\
\hline
\end{tabular}

${ }^{a} \rho_{c}$ - electron density at BCP, a.u.; $\varepsilon$ - bond ellipticity; $\nabla^{2}\left(\rho_{c}\right)$ - Laplacian of Rho = trace of hessian of Rho; $V=$ virial field $=$ potential energy density = trace of stress tensor; $G=$ Lagrangian form of kinetic energy density; $K=$ Hamiltonian form of kinetic energy density $=L$ $=\mathrm{K}-\mathrm{G}=$ Lagrangian density $=(-1 / 4) \nabla^{2}\left(\rho_{\mathrm{c}}\right) ; \mathrm{DI}=$ delocalization index $=$ bond order . 
Analysis of complexes 7 and 8 using EDA method at ADF/BP86-D3/TZP calculations level (Table S10) revealed that total interaction energies $E_{\text {int }}$ of $\mathrm{Fe}-\mathrm{C}$ bonds are not sensitive to the position of heterocyclic ring in a sharp contrast to those in $\mathrm{NHC}$ and $a \mathrm{NHC}$ derivatives. The values of $\mathrm{E}_{\text {int }}$ in $\sigma$ imidazolyl complexes are $70-90 \%$ greater than those found for $\mathrm{Fe}-\mathrm{NHC}$ and $\mathrm{Fe}-\mathrm{aNHC}$ bonds in cationic species [4-6] $]^{+}$. This effect is much less pronounced for $\mathrm{Fe}-\mathrm{aNHC}$ bond in a globally neutral dIMes complex 3, but still remains significant (10-12\%). The overall $\mathrm{Fe}-\mathrm{C}$ bond energy in $\sigma$-methyl complex $\mathbf{9}$ is ca. $10 \%$ greater than for the corresponding $\sigma$-imidazolyl species 8 and $\mathbf{9}$. The largest portion of this difference comes from the increase of the electrostatic attraction and repulsion interactions (Table S10). This situation can be more conveniently illustrated by comparing the $\mathrm{E}_{\text {steric }}$ energy contribution in Fe-carbene bonds being mostly close to zero $\left(0.2\right.$ to $\left.-5.6 \mathrm{kcal} \mathrm{mol}^{-1}\right)$ with highly negative values found in the model $\sigma$-complexes 7-9 $\left(-80.5\right.$ to $\left.-98.3 \mathrm{kcal} \mathrm{mol}^{-1}\right)$. The values of $\Delta \mathrm{E}_{\text {steric }}$ between $\mathrm{Fe}-\mathrm{aNHC}$ bonds in complexes $\mathbf{3 a}$ and $\mathbf{3 b}$ and $\mathrm{Fe}-\mathrm{C}$ bonds in $\sigma$-imidazolyl species 7-8 (23.9-28.3 kcal mol$\left.{ }^{-1}\right)$ is more significant than between the later and $\sigma$-methyl complex 9 (14.3$\left.17.8 \mathrm{kcal} \mathrm{mol}^{-1}\right)$. In addition, ETS-NOCV analysis data show the increased contribution of $\sigma$-donation component in orbital energy of $\mathrm{Fe}-\mathrm{C}$ interaction described by $\mathrm{E}_{\sigma} / \mathrm{E}_{\text {orb }}$ ratio going from $0.60-0.63$ and 0.68-0.69 for $\mathrm{Fe}-\mathrm{NHC}$ and $\mathrm{Fe}-\mathrm{aNHC}$ bonds, respectively, to $0.74-75$ for $\mathrm{Fe}-\mathrm{C}$ bond in $\sigma$-heteroaryl complexes. Similar trend in $\mathrm{E}_{\sigma} / \mathrm{E}_{\text {orb }}$ ratio $(0.74-0.75$ vs. 0.81$)$ was also noticed between $\mathrm{Fe}(\mathrm{II}) \sigma-$ imidazolyl (7 and 8 ) and $\sigma$-methyl (9) complexes. These results show that the $\mathrm{Fe}-\mathrm{aNHC}$ interaction in dlMes complexes $\mathbf{3}$ and $\mathbf{5}^{+}$can be better described as dative bond, which differs significantly from mainly electrostatically-driven $\mathrm{Fe}-\mathrm{C}$ bonds with pronounced $\sigma$-character in model complexes 7-9. Though according to these data we can not completely rule out partial contribution of LX resonance form (Scheme S1) in complex 3, our study clearly demonstrates that the most chemically reasonable representation of NHDC complexes includes dicarbene structure with delocalized negative charge within the heterocyclic core.

Table S10. The comparison of ETS-NOCV data for Fe-C bonding in optimized Fe(II) $\sigma$-complexes 7-9 with those of Fe-NHC bonding in IMes, alMes and dlMes complexes (ADF-BP86-D3-TZP, energies are given in $\mathrm{kcal} \mathrm{mol}^{-1}$ ).

\begin{tabular}{|c|c|c|c|c|c|c|c|c|c|c|c|c|}
\hline Complex & $6 \mathrm{a}^{+}$ & $6 b^{+}$ & $4^{+}$ & $\begin{array}{c}3 a \\
M-a N H C\end{array}$ & $\begin{array}{c}3 \mathbf{b} \\
\mathrm{M}-\mathrm{aNHC}\end{array}$ & $\begin{array}{c}\mathbf{5} \mathbf{a}^{+} \\
\mathrm{M}-\mathrm{NHC}\end{array}$ & $\begin{array}{c}5 \mathbf{a}^{+} \\
\mathrm{M}-\mathrm{aNHC}\end{array}$ & $\begin{array}{c}5 b^{+} \\
M-N H C\end{array}$ & $\begin{array}{c}5 \mathbf{b}^{+} \\
M-a N H C\end{array}$ & 7 & 8 & 9 \\
\hline$E_{\text {Pauli }}$ & 144.1 & 145.1 & 155.3 & 172.7 & 173.0 & 141.7 & 150.5 & 145.5 & 149.9 & 194.9 & 194.7 & 195.2 \\
\hline Eelst & -145.3 & -144.9 & -160.9 & -229.3 & -228.7 & -145.6 & -154.8 & -146.5 & -153.2 & -278.9 & -275.2 & -293.5 \\
\hline$E_{\text {disp }}$ & -23.8 & -24.1 & -16.0 & -17.4 & -17.4 & -24.7 & -17.2 & -24.9 & -17.4 & -9.8 & -10.1 & -5.7 \\
\hline$E_{\text {int }}$ & -112.7 & -113.1 & -117.5 & -188.9 & -188.6 & -120.1 & -125.2 & -120.3 & -124.6 & -212.4 & -207.7 & -231.1 \\
\hline$E_{\text {orb }}$ & -87.8 & -89.2 & -95.9 & -114.9 & -115.4 & -91.6 & -103.6 & -94.4 & -103.8 & -118.7 & -117.1 & -127.0 \\
\hline$E_{\text {steric }}=E_{\text {Pauli }}+E_{\text {elst }}$ & -1.2 & 0.2 & -5.6 & -56.6 & -55.7 & -3.9 & -4.3 & -1.0 & -3.3 & -84.0 & -80.5 & -98.3 \\
\hline$E_{\text {ort }} / E_{\text {int }}$ & 0.78 & 0.79 & 0.82 & 0.61 & 0.61 & 0.76 & 0.83 & 0.78 & 0.83 & 0.56 & 0.56 & 0.55 \\
\hline$E_{\text {elstat }} / E_{\text {int }}$ & 1.29 & 1.28 & 1.37 & 1.21 & 1.21 & 1.21 & 1.24 & 1.22 & 1.23 & 1.31 & 1.32 & 1.37 \\
\hline $\mathrm{E}_{\sigma}(\mathrm{C} \rightarrow \mathrm{M})$ & -54.3 & -53.9 & -64.8 & -78.2 & -77.9 & -57.3 & -71.5 & -57.5 & -71.3 & -87.6 & -87.7 & -103.3 \\
\hline$E_{\sigma}(C \rightarrow M) / E_{o r b}$ & 0.62 & 0.60 & 0.68 & 0.68 & 0.68 & 0.63 & 0.69 & 0.61 & 0.69 & 0.74 & 0.75 & 0.81 \\
\hline $\mathrm{E}_{\Pi \perp}(\mathrm{M} \rightarrow \mathrm{C})$ & -9.6 & -9.8 & -9.1 & -8.7 & -8.9 & -9.2 & -8.7 & -9.9 & -9.0 & -8.1 & -7.9 & $-4.1^{b}$ \\
\hline$E_{\pi \perp}(M \rightarrow C) / E_{o r b}$ & 0.11 & 0.11 & 0.09 & 0.08 & 0.08 & 0.10 & 0.08 & 0.10 & 0.09 & 0.07 & 0.07 & 0.03 \\
\hline $\mathrm{E}_{\pi}(\mathrm{M} \rightarrow \mathrm{C})^{a}$ & -3.8 & -6.3 & -4.4 & $-9.5^{]}$ & -7.5 & -4.1 & -3.2 & -6.5 & -3.4 & -7.3 & -6.9 & $-3.1^{b}$ \\
\hline
\end{tabular}

${ }^{a}$ The sum of energies for other $\pi$ interactions. ${ }^{b}$ The interaction energy corresponding to the overlap between metal orbitals and $\sigma^{\star}$-orbitals of $\mathrm{C}-\mathrm{H}$ bonds in the methyl group. 


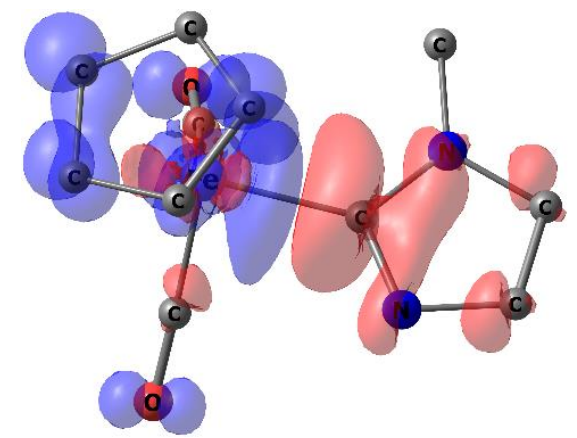

NOCV1, $E=-87.6(\sigma \mathrm{C} \rightarrow \mathrm{M})$

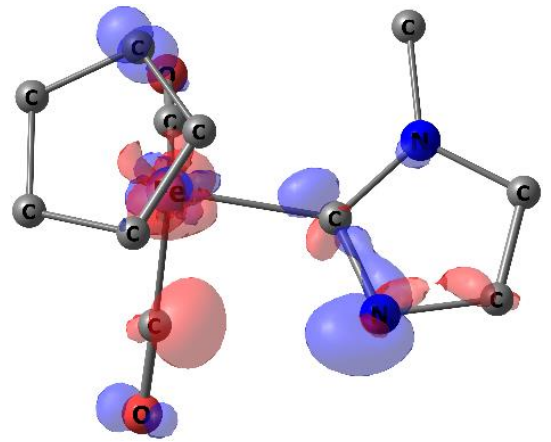

NOCV4, $E=-2.3$ (other $M \rightarrow C$ )

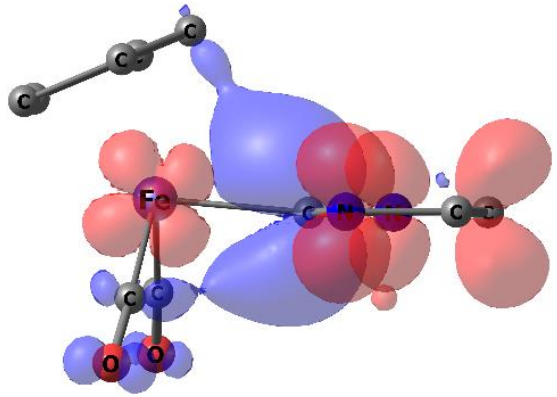

NOCV2, $E=-8.1(\pi 1 \mathrm{M} \rightarrow \mathrm{C})$

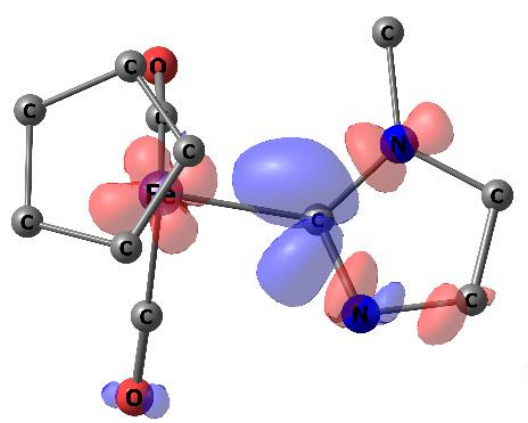

NocV5, $E=-2.7(\pi 2 M \rightarrow C)$

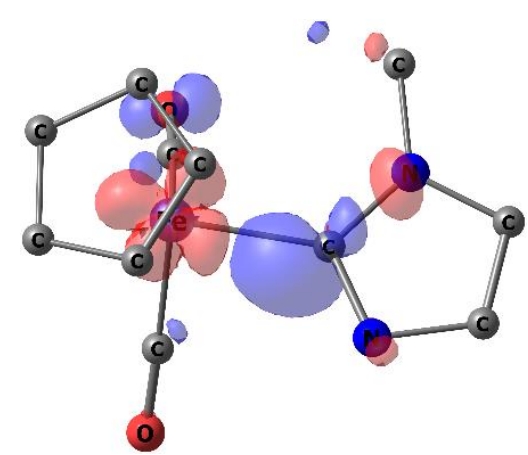

NOCV3, $E=-4.6(\pi 2 M \rightarrow C)$

Figure S20. NOCV deformation electron density for complex 7 (isosurface at 0.003 and 0.001 a.u. for NOCV1 and NOCV2-5, respectively) and associated energies in kcal mol-1. Red areas correspond to the depletion and blue to the concentration of electron density. Hydrogen atoms are omitted for clarity.

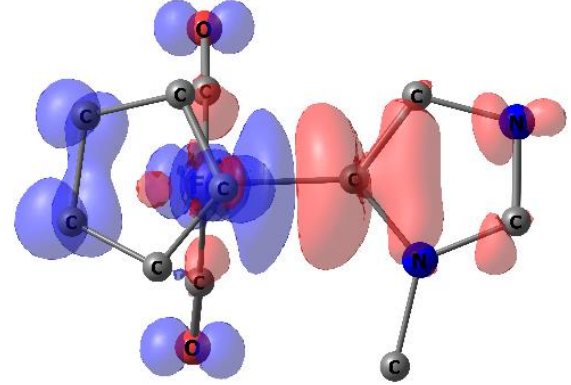

NOCV1, $E=-87.7(\sigma C \rightarrow M)$

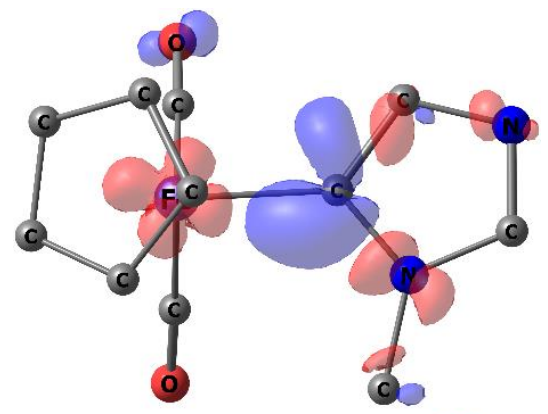

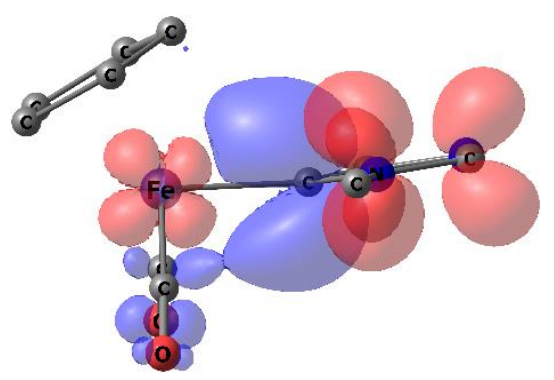

NOCV2, $E=-7.9(\pi 1 \mathrm{M} \rightarrow \mathrm{C})$

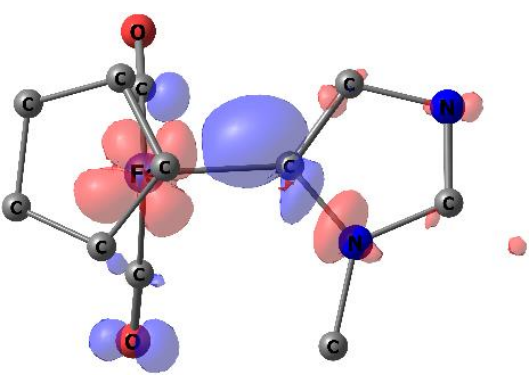

NOCV3, $E=-4.1(\pi 2 M \rightarrow C)$

NOCV4, $E=-2.8(\pi 2 \mathrm{M} \rightarrow \mathrm{C})$

Figure S21. NOCV deformation electron density for complex 8 (isosurface at 0.003 and 0.001 a.u. for NOCV1 and NOCV2-4, respectively) and associated energies in $\mathrm{kcal} \mathrm{mol}^{-1}$. Red areas correspond to the depletion and blue to the concentration of electron density. Hydrogen atoms are omitted for clarity. 


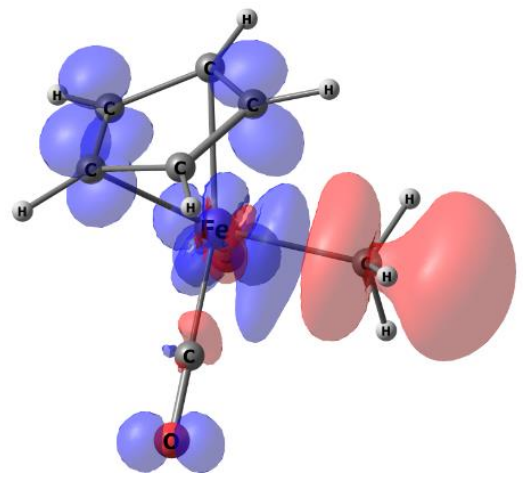

NOCV1, $E=-103.3(\sigma C \rightarrow M)$

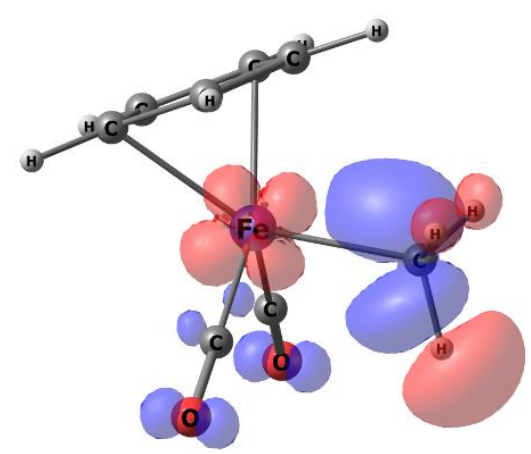

NOCV2, $E=-4.1(\pi 1 M \rightarrow C-H)$

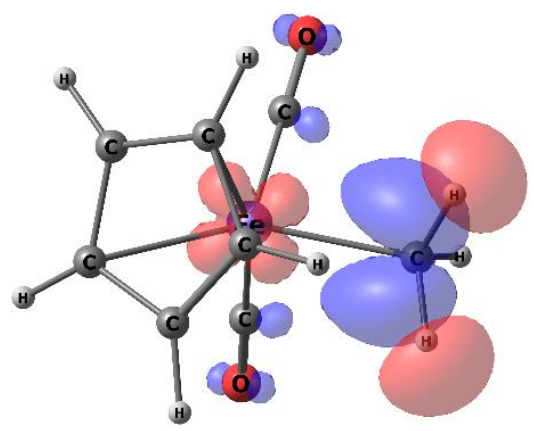

NocV3, $E=-3.1(\pi 2 M \rightarrow C-H)$

Figure S22. NOCV deformation electron density for complex 9 (isosurface at 0.003 and 0.001 a.u. for NOCV1 and NOCV2-3, respectively) and associated energies in $\mathrm{kcal} \mathrm{mol}^{-1}$. Red areas correspond to the depletion and blue to the concentration of electron density. 

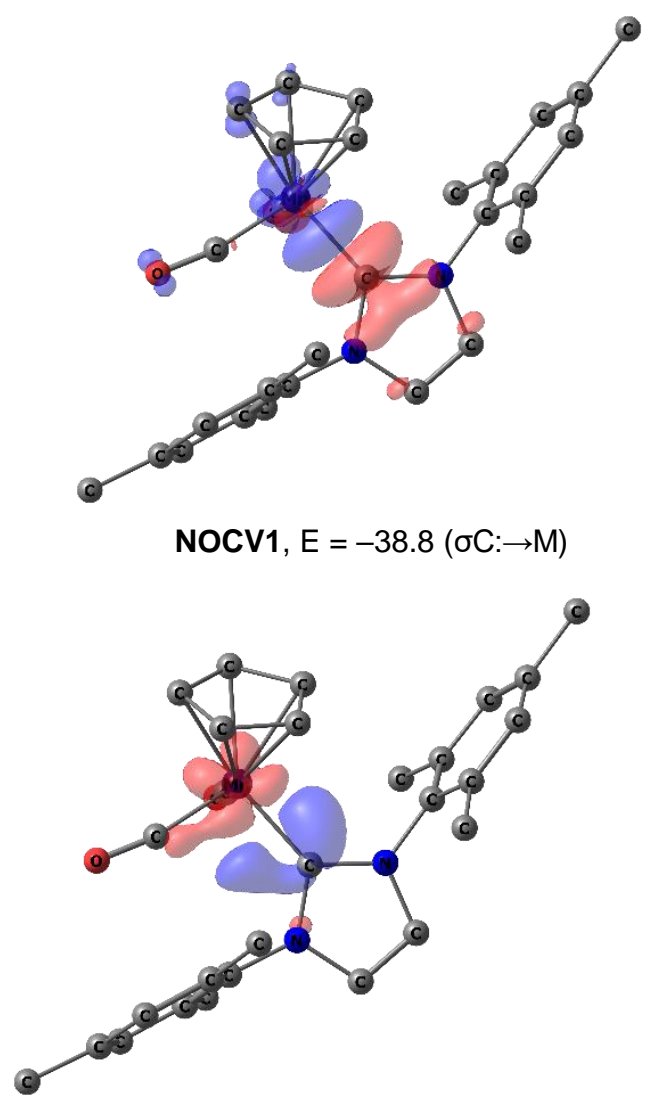

NocV3, $E=-5.4\left(\pi_{\|} M \rightarrow C:\right)$

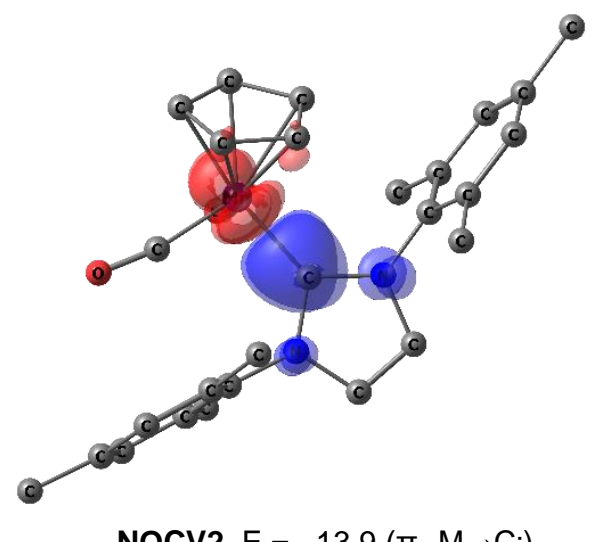

NOCV2, $E=-13.9\left(\pi_{\perp} M \rightarrow C:\right)$

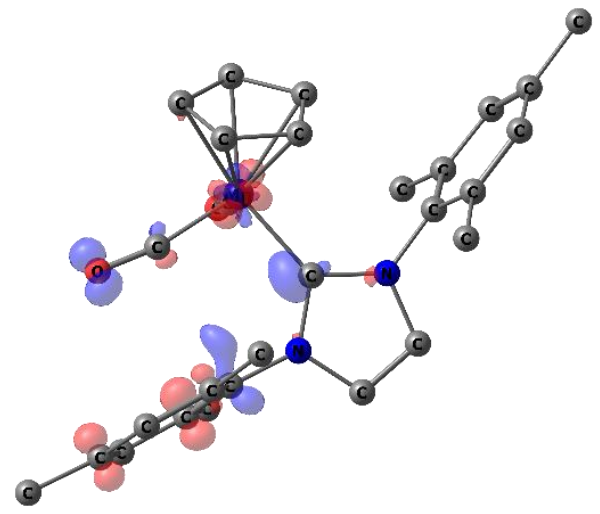

NOCV4, $E=-1.5\left(\pi(C=C) \rightarrow \pi^{*}(C O)\right)$

Figure S23. NOCV deformation electron density for complex $\mathbf{1 b}$ (isosurface at 0.003 and 0.001 a.u. for NOCV1 and NOCV2-4, respectively) and associated energies in kcal mol${ }^{-1}$. Red areas correspond to the depletion and blue to the concentration of electron density. Hydrogen atoms are omitted for clarity.

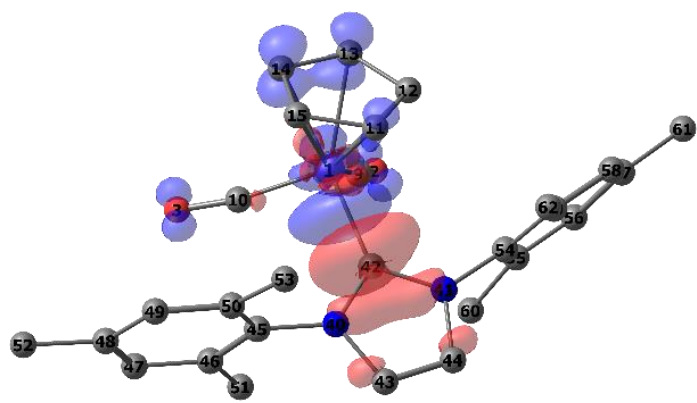

NOCV1, $E=-54.3(\sigma \mathrm{C}: \rightarrow \mathrm{M})$

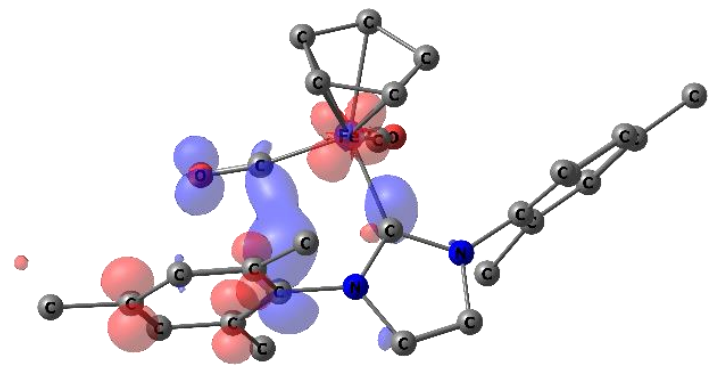

NoCV3, $E=-3.3\left(\pi(C=C) \rightarrow \pi^{*}(C O)\right)$

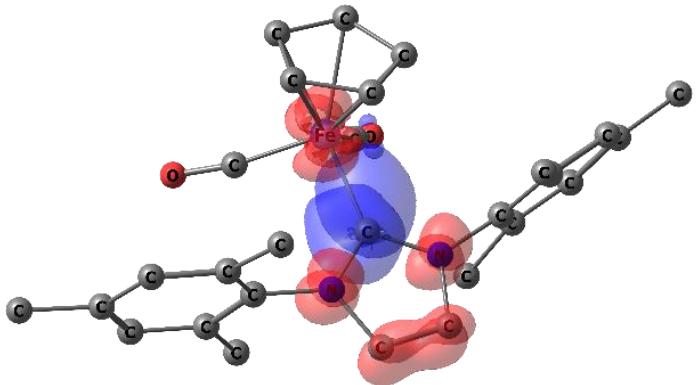

NOCV2, $E=-9.6(\pi \perp M \rightarrow C:)$

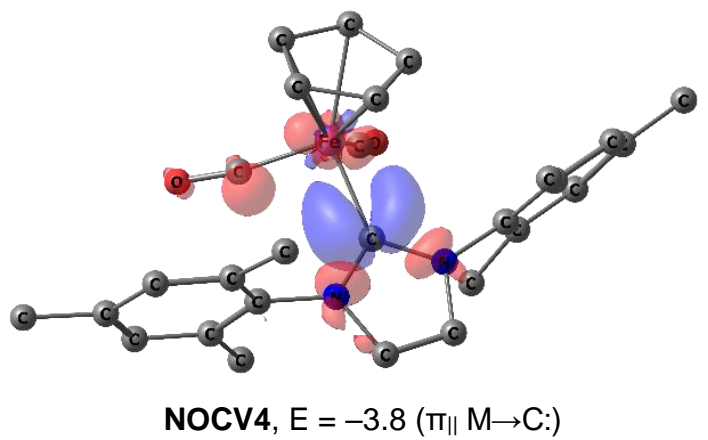

Figure S24. NOCV deformation electron density for complex $6 \mathbf{a}^{+}$(isosurface at 0.003 and 0.001 a.u. for NOCV1 and NOCV2-4, respectively) and associated energies in $\mathrm{kcal} \mathrm{mol}^{-1}$. Red areas correspond to the depletion and blue to the concentration of electron density. Hydrogen atoms are omitted for clarity. 

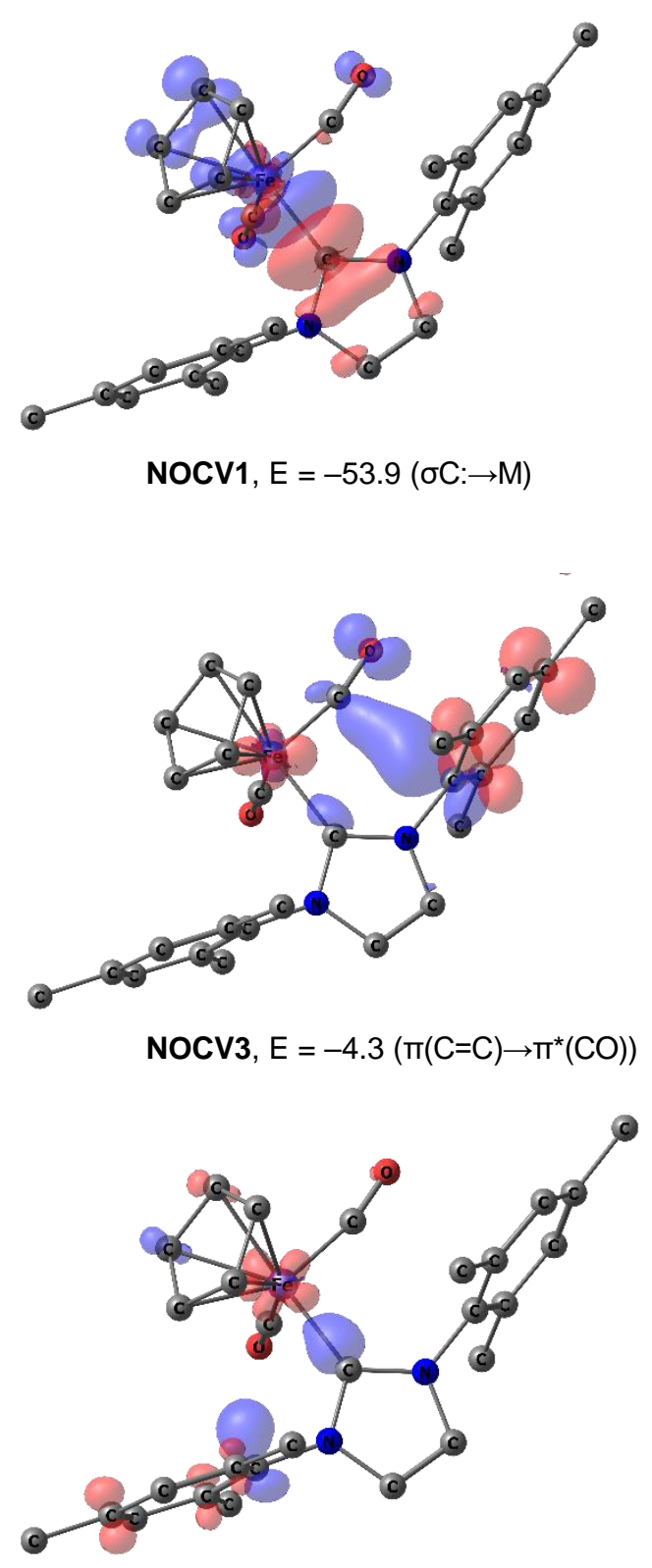

$$
\text { NocV5, } E=-2.2(\pi \mathrm{M} \rightarrow \mathrm{C}:)
$$
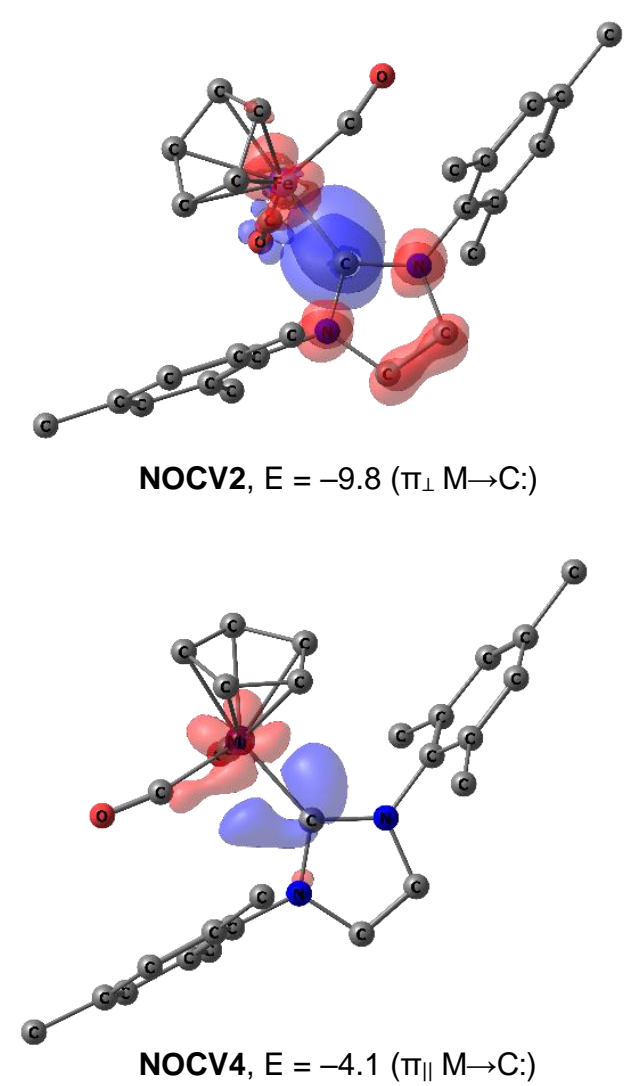

Figure S25. NOCV deformation electron density for complex $6 \mathbf{b}^{+}$(isosurface at 0.003 and 0.001 a.u. for NOCV1 and NOCV2-5, respectively) and associated energies in $\mathrm{kcal} \mathrm{mol}^{-1}$. Red areas correspond to the depletion and blue to the concentration of electron density. Hydrogen atoms are omitted for clarity. 


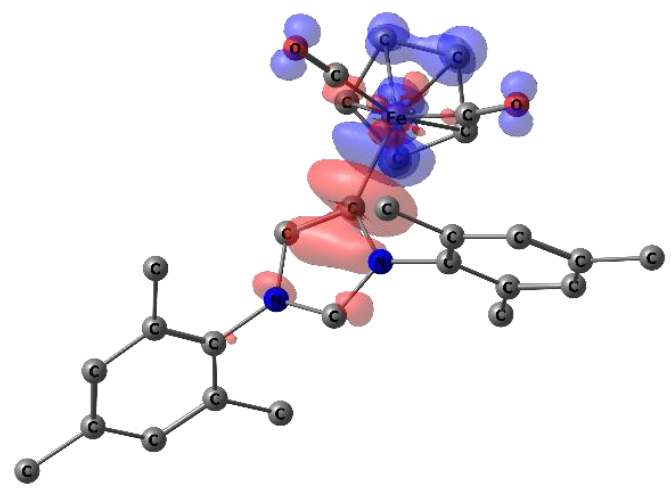

NOCV1, $E=-64.8(\sigma \mathrm{C}: \rightarrow \mathrm{M})$

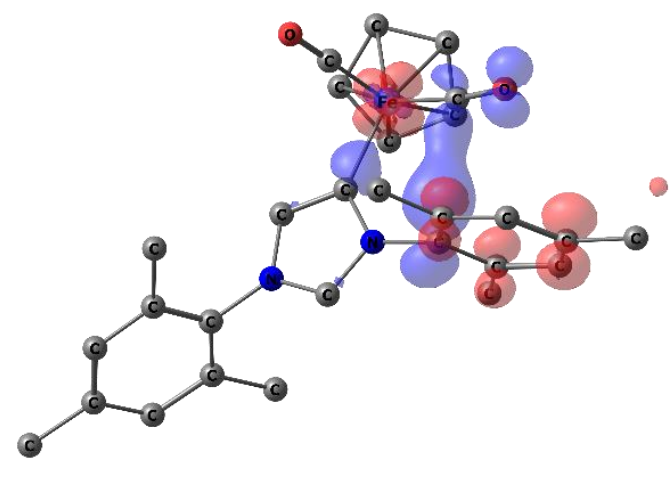

NOCV3, $E=-3.7\left(\pi(C=C) \rightarrow \pi^{*}(C O)\right)$

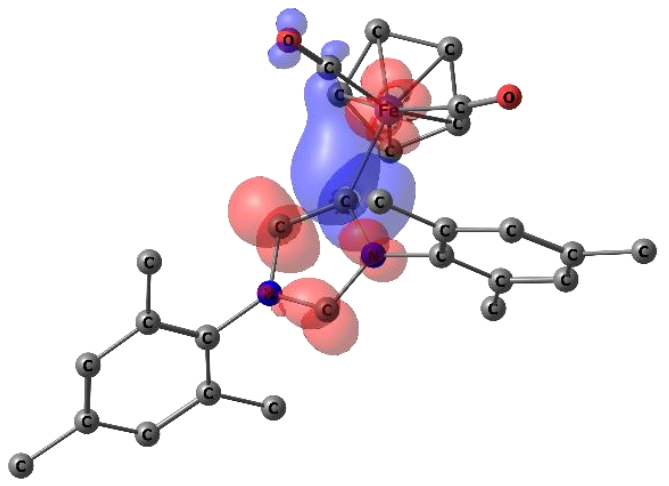

NOCV2, $E=-9.1\left(\pi_{\perp} M \rightarrow C:\right)$

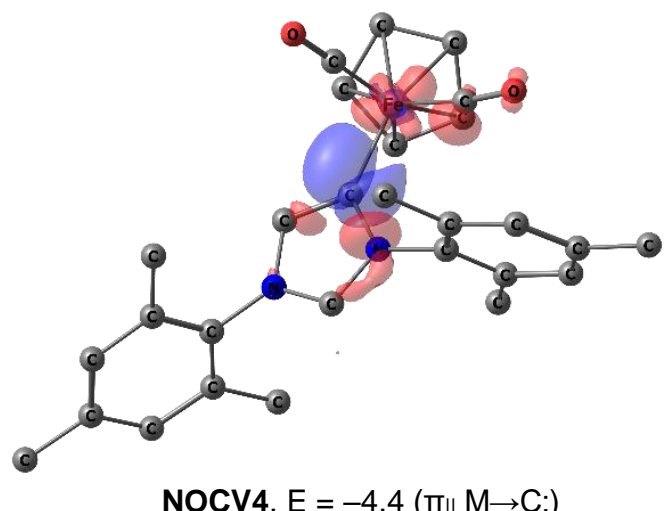

NOCV4, $E=-4.4(\pi|| M \rightarrow C:)$

Figure S26. NOCV deformation electron density for complex 4+ (isosurface at 0.003 and 0.001 a.u. for NOCV1 and NOCV2-4, respectively) and associated energies in $\mathrm{kcal} \mathrm{mol}^{-1}$. Red areas correspond to the depletion and blue to the concentration of electron density. Hydrogen atoms are omitted for clarity. 


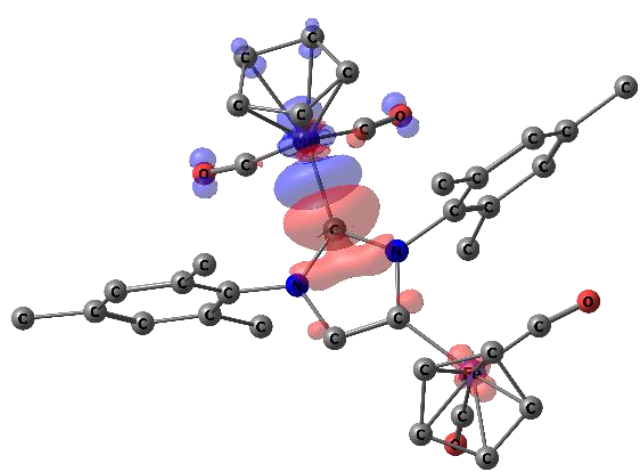

NOCV1, $E=-38.6(\sigma C: \rightarrow M)$

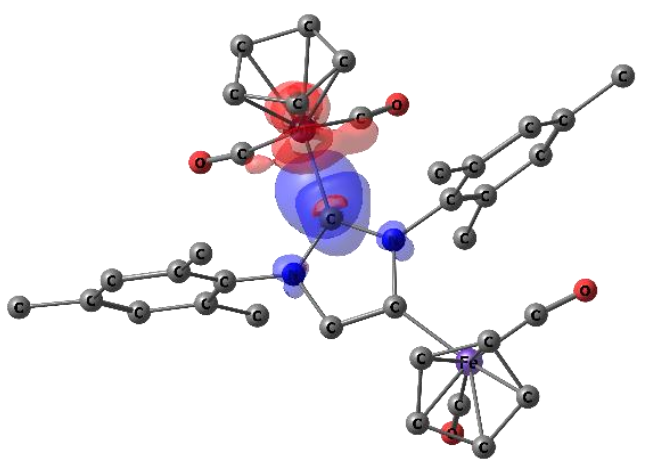

NOCV2, $E=-11.2\left(\pi_{\perp} M \rightarrow C:\right)$

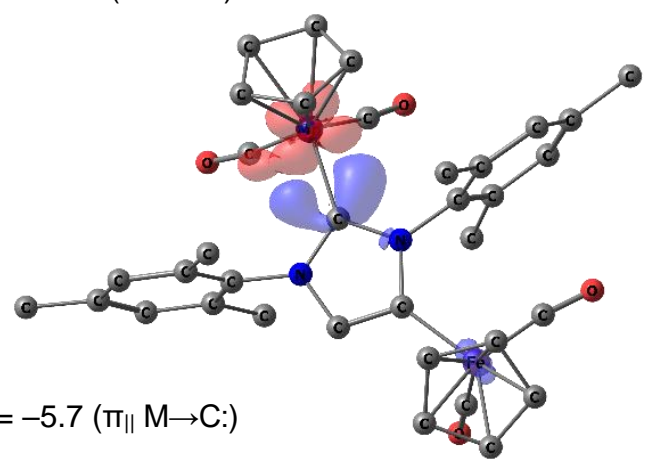

NOCV3, $E=-5.7(\pi \| M \rightarrow C:)$

Figure S27. NOCV deformation electron density for $\mathrm{Mn}-\mathrm{NHC}$ bond in complex $\mathbf{3 a}$ (isosurface at 0.003 and 0.001 a.u. for NOCV1 and NOCV2-3, respectively) and associated energies in kcal mol-1. Red areas correspond to the depletion and blue to the concentration of electron density. Hydrogen atoms of aryls are omitted for clarity.
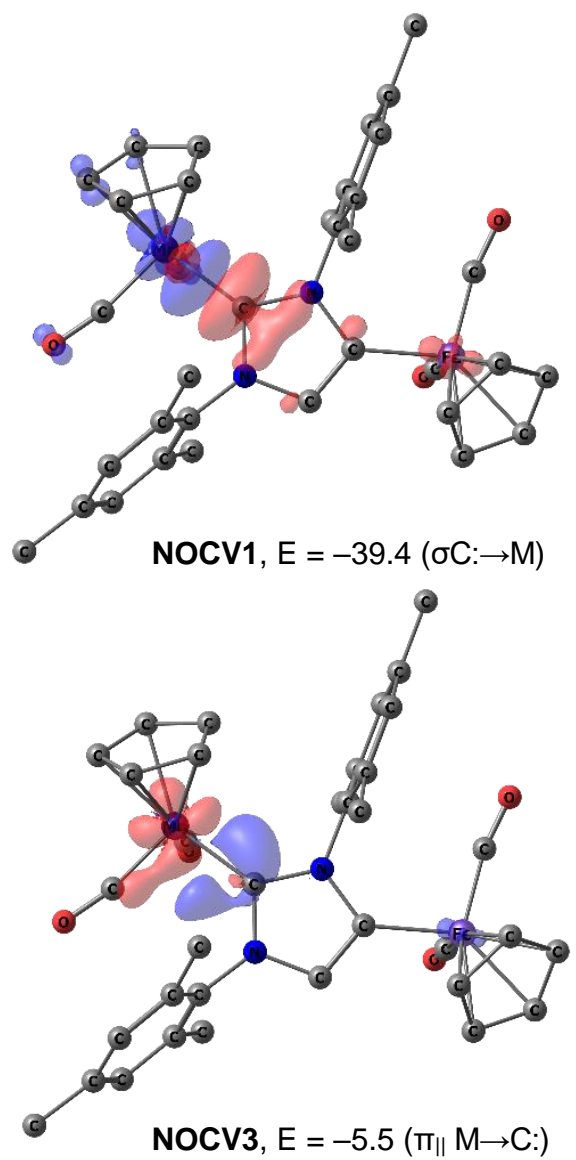
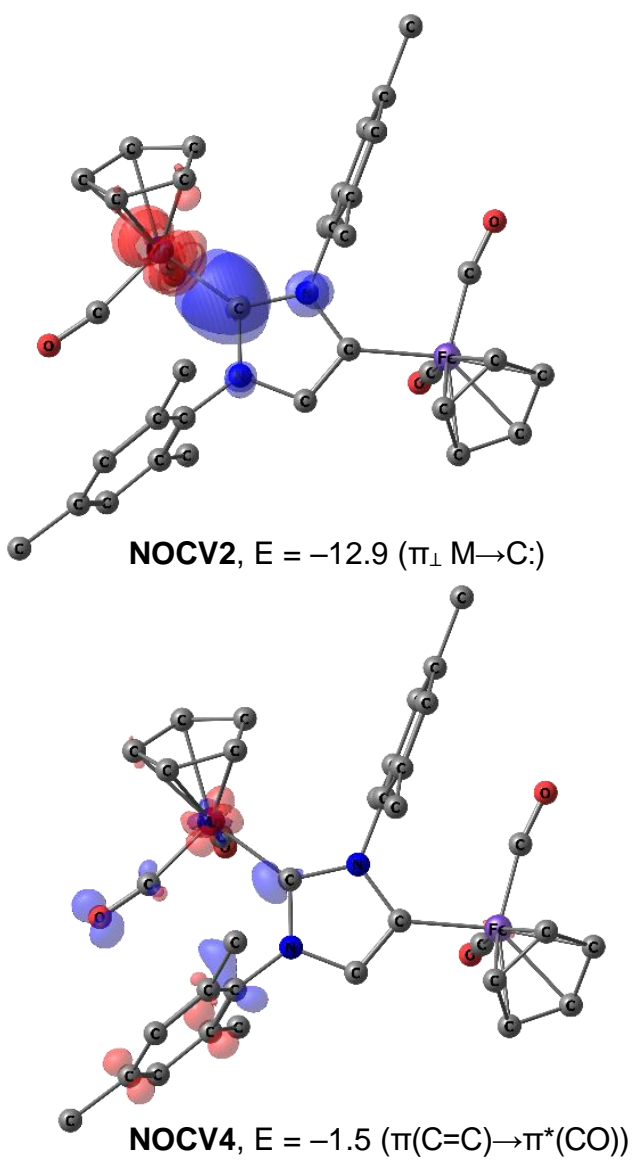

Figure S28. NOCV deformation electron density for Mn-NHC bond in complex $\mathbf{3 b}$ (isosurface at 0.003 and 0.001 a.u. for NOCV1 and NOCV2-4, respectively) and associated energies in $\mathrm{kcal} \cdot \mathrm{mol}^{-1}$. Red areas correspond to the depletion and blue to the concentration of electron density. Hydrogen atoms of aryls are omitted for clarity. 


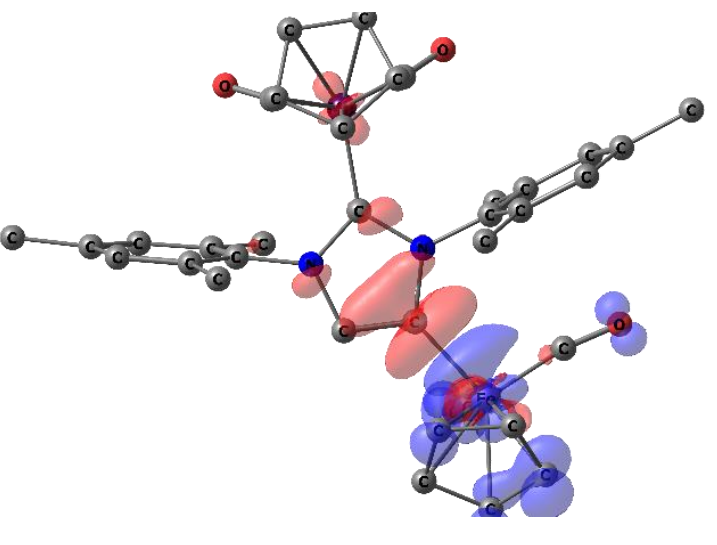

NOCV1, $E=-78.2(\sigma C: \rightarrow M)$

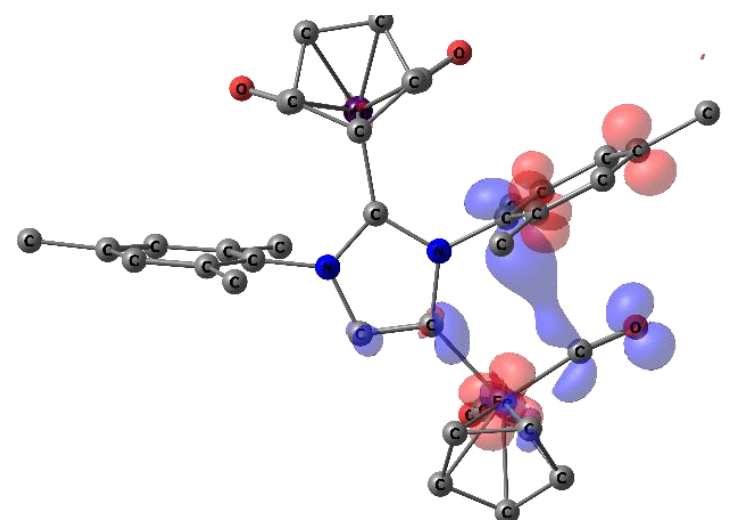

NocV3, $E=-4.1\left(\pi(C=C) \rightarrow \pi^{\star}(C O)\right)$

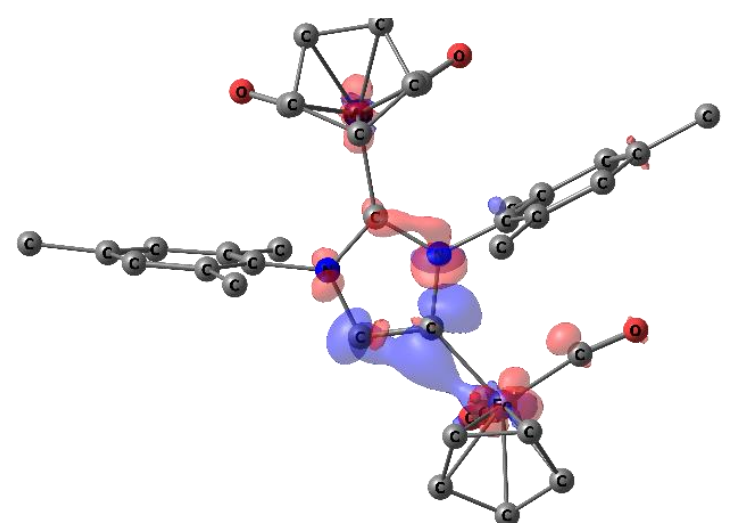

NOCV5, $E=-2.8\left(\pi 1_{\|} M \rightarrow C:\right)$

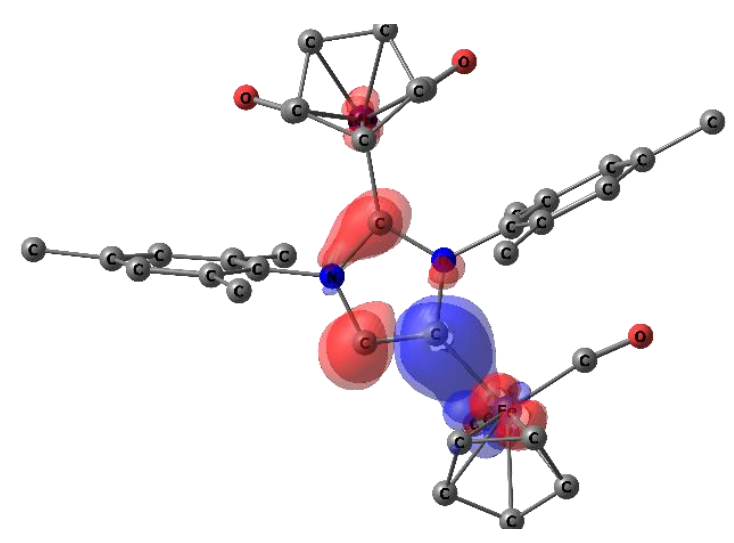

NOCV2, $E=-8.7\left(\pi_{\perp} M \rightarrow C:\right)$

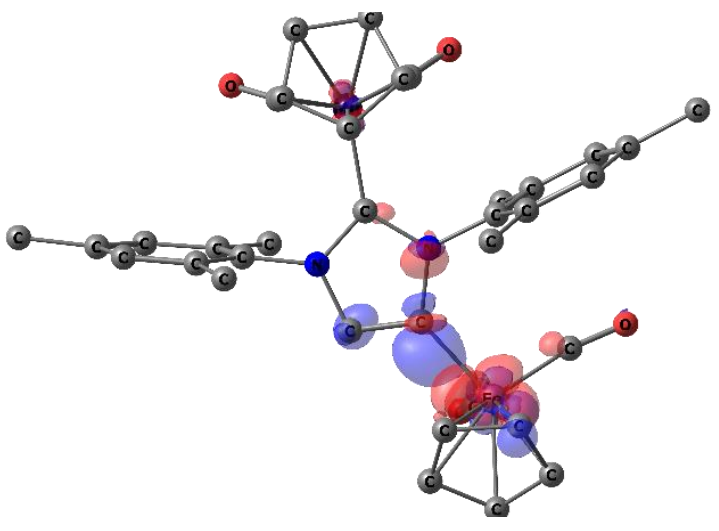

NoCV4, $E=-4.2(\pi \mathrm{M} \rightarrow \mathrm{C}:)$

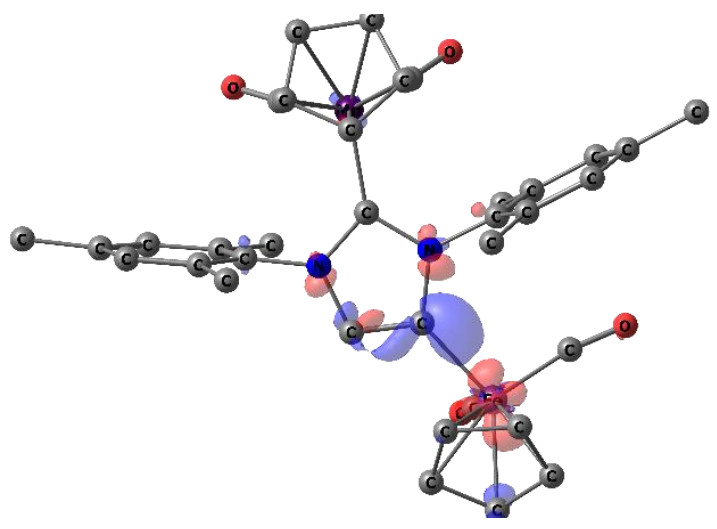

NocV6, $E=-2.5(\pi 2 \| M \rightarrow C:)$

Figure S29. NOCV deformation electron density for Fe-aNHC bond in complex $\mathbf{3 a}$ (isosurface at 0.003 and 0.001 a.u. for NOCV1 and NOCV2-6, respectively) and associated energies in $\mathrm{kcal} \mathrm{mol}{ }^{-1}$. Red areas correspond to the depletion and blue to the concentration of electron density. Hydrogen atoms of aryls are omitted for clarity. 

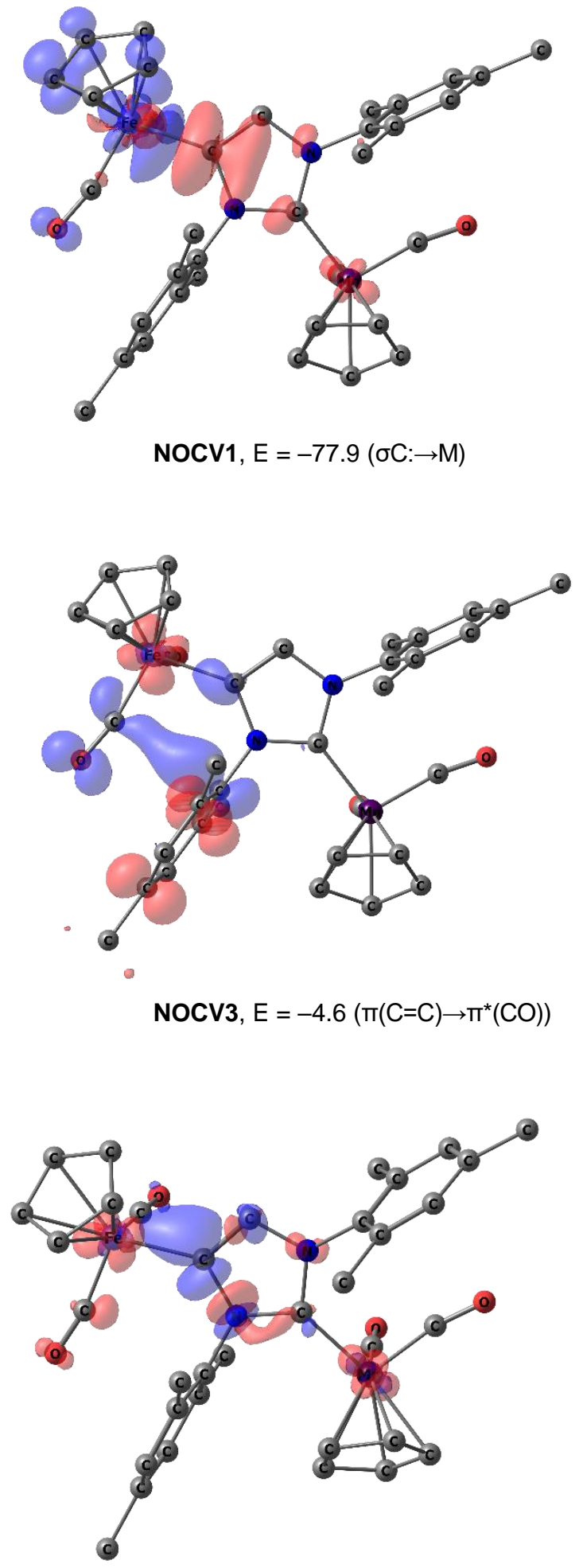

NoCV5, $E=-3.5(\pi|| M \rightarrow C:)$

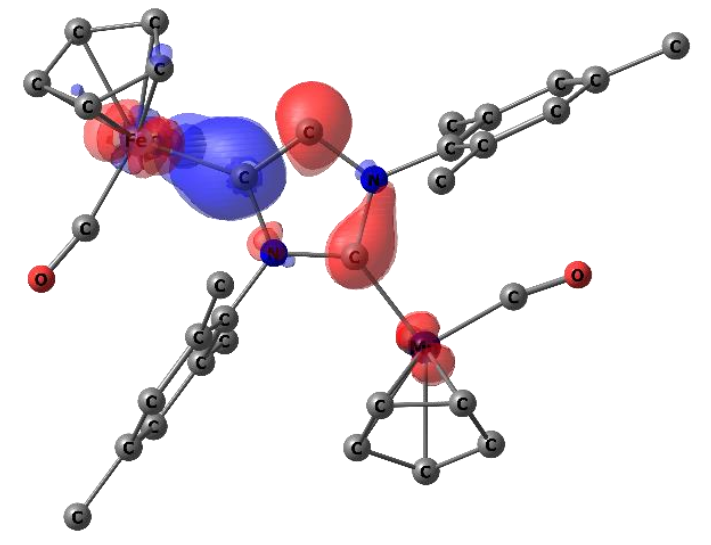

NoCV2, $E=-8.9\left(\pi_{\perp} M \rightarrow C:\right)$

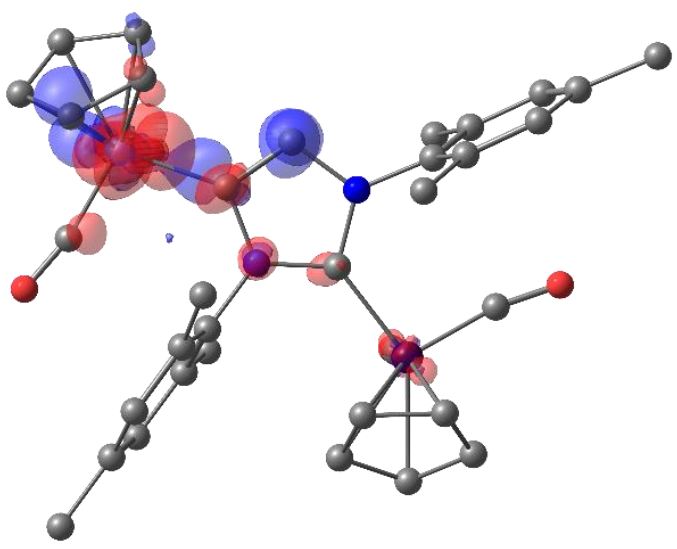

NOCV4, $E=-4.0(\pi \mathrm{M} \rightarrow \mathrm{C}:)$

Figure S30. NOCV deformation electron density for $\mathrm{Fe}-\mathrm{aNHC}$ bond in complex $\mathbf{3 b}$ (isosurface at 0.003 and 0.001 a.u. for NOCV1 and NOCV2-5, respectively) and associated energies in $\mathrm{kcal} \mathrm{mol}{ }^{-1}$. Red areas correspond to the depletion and blue to the concentration of electron density. Hydrogen atoms of aryls are omitted for clarity. 


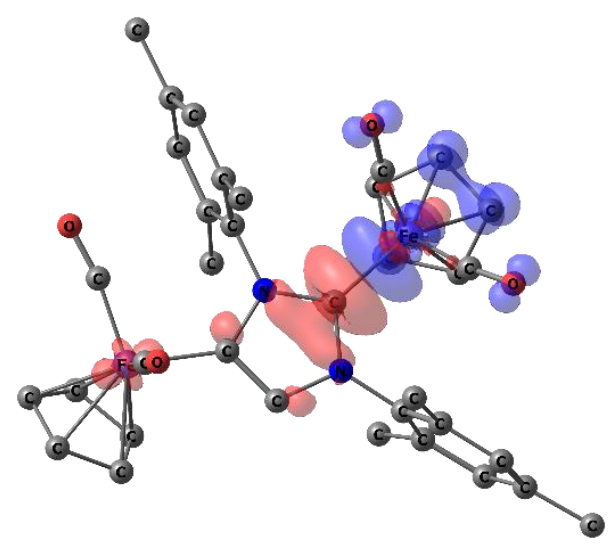

NocV1, $E=-57.3(\sigma \mathrm{C}: \rightarrow \mathrm{M})$

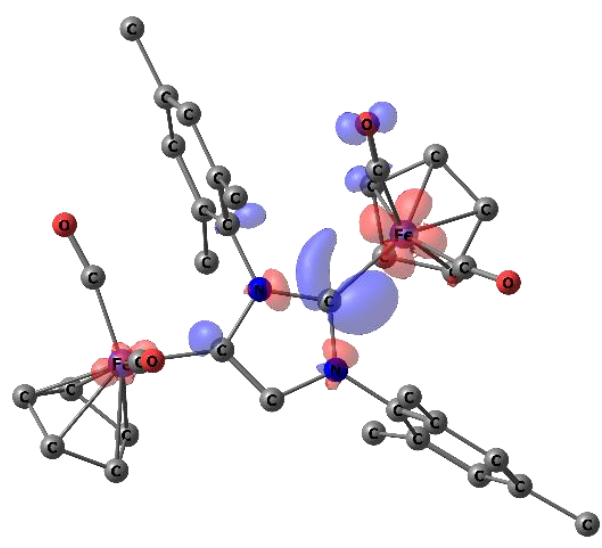

NocV3, $E=-4.1\left(\pi_{\|} M \rightarrow C:\right)$

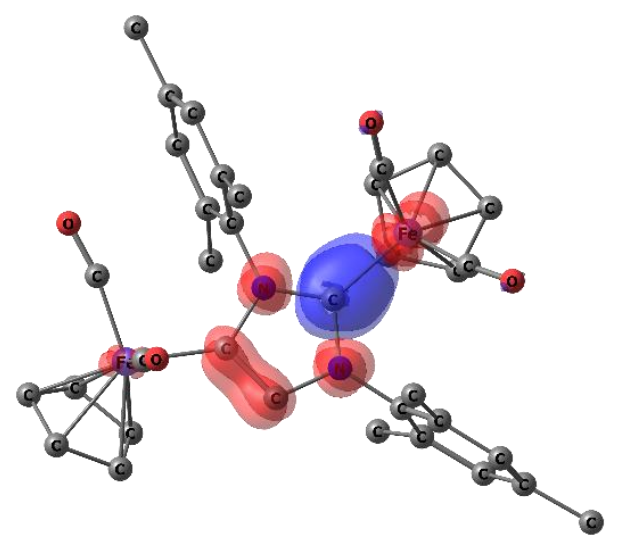

NOCV2, $E=-9.2(\pi \perp M \rightarrow C:)$

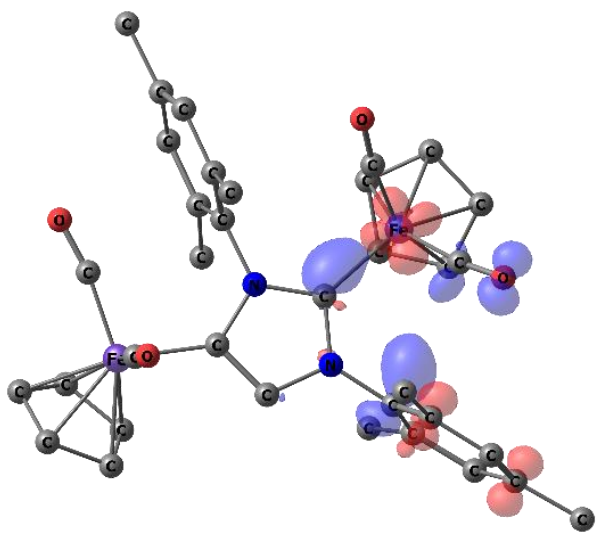

NoCV4, $E=-3.2\left(\pi(C=C) \rightarrow \pi^{*}(C O)\right)$

Figure S31. NOCV deformation electron density for Fe-NHC bond in complex $5 \mathbf{a}^{+}$(isosurface at 0.003 and 0.001 a.u. for NOCV1 and NOCV2-4, respectively) and associated energies in $\mathrm{kcal} \mathrm{mol}{ }^{-1}$. Red areas correspond to the depletion and blue to the concentration of electron density. Hydrogen atoms of aryls are omitted for clarity. 


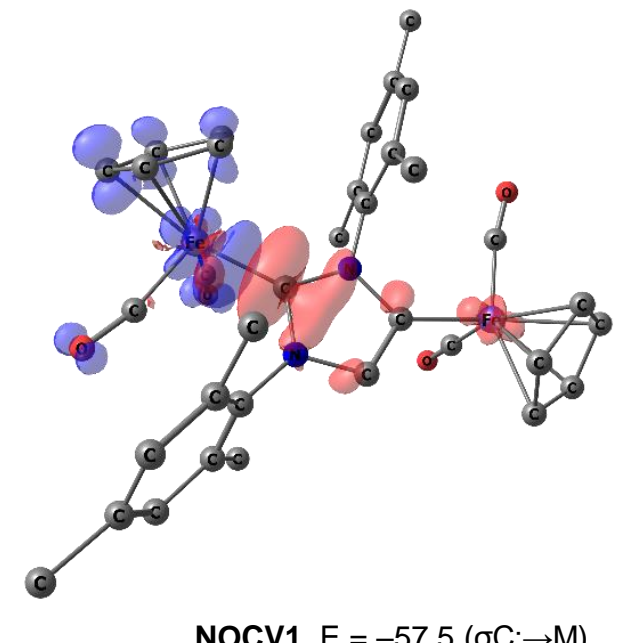

NOCV1, $E=-57.5(\sigma C: \rightarrow M)$

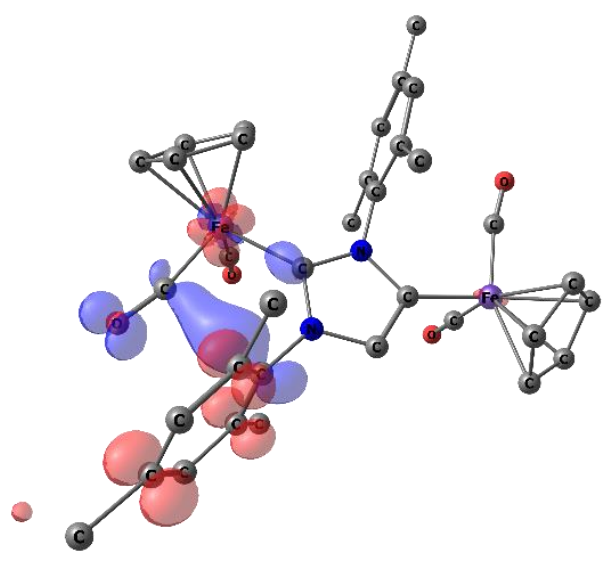

Nocv3, $E=-4.2\left(\pi(C=C) \rightarrow \pi^{*}(C O)\right)$
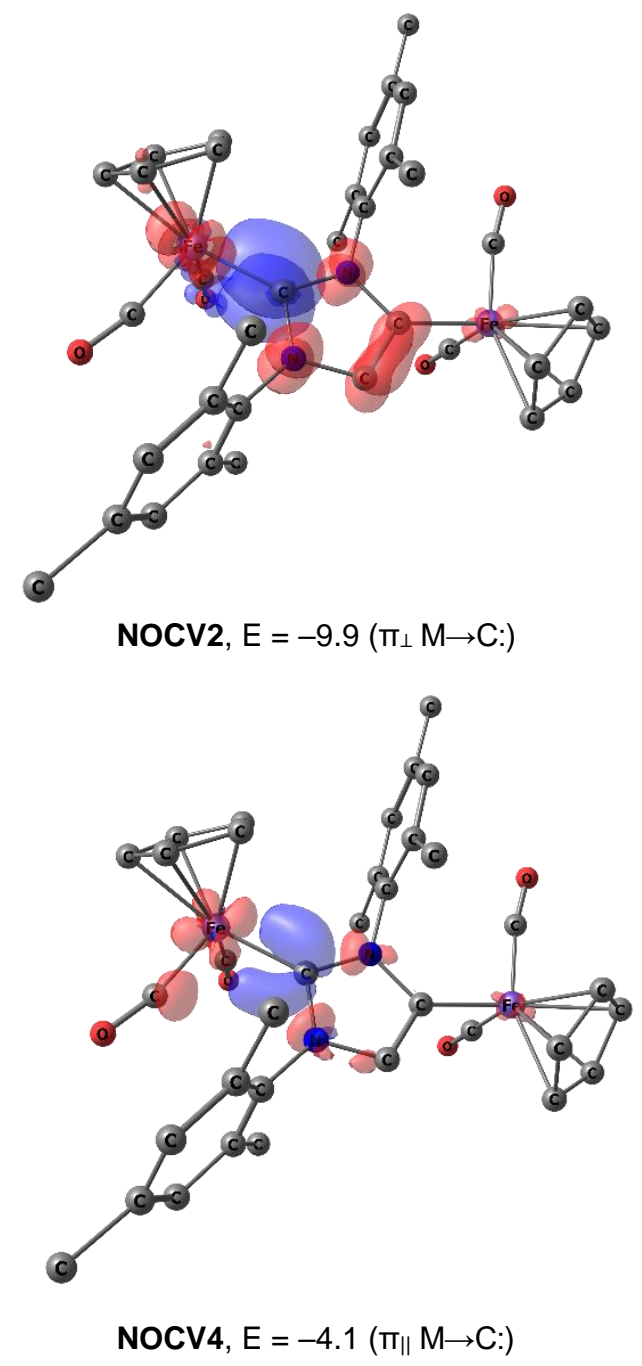

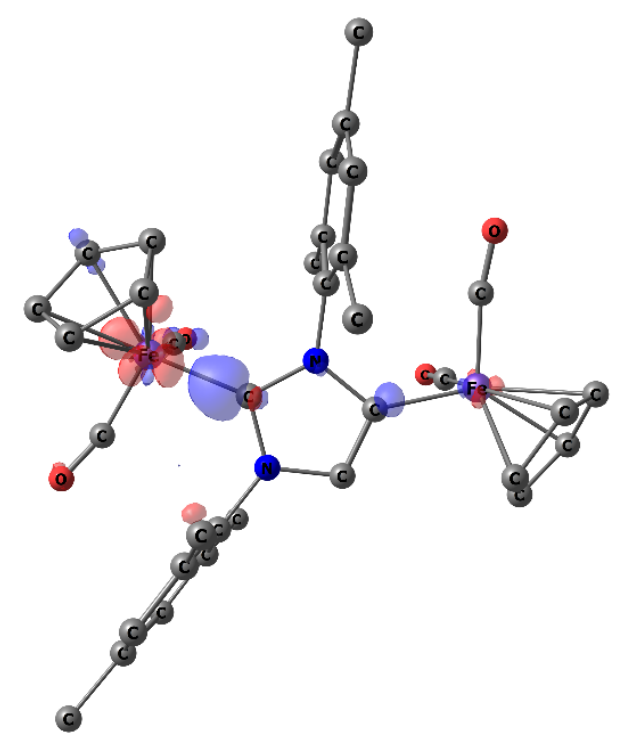

NoCV5, $E=-2.4(\pi M \rightarrow C:)$

Figure S32. NOCV deformation electron density for Fe-NHC bond in complex $\mathbf{5} \mathbf{b}^{+}$(isosurface at 0.003 and 0.001 a.u. for NOCV1 and NOCV2-5, respectively) and associated energies in $\mathrm{kcal} \mathrm{mol}{ }^{-1}$. Red areas correspond to the depletion and blue to the concentration of electron density. Hydrogen atoms of aryls are omitted for clarity. 


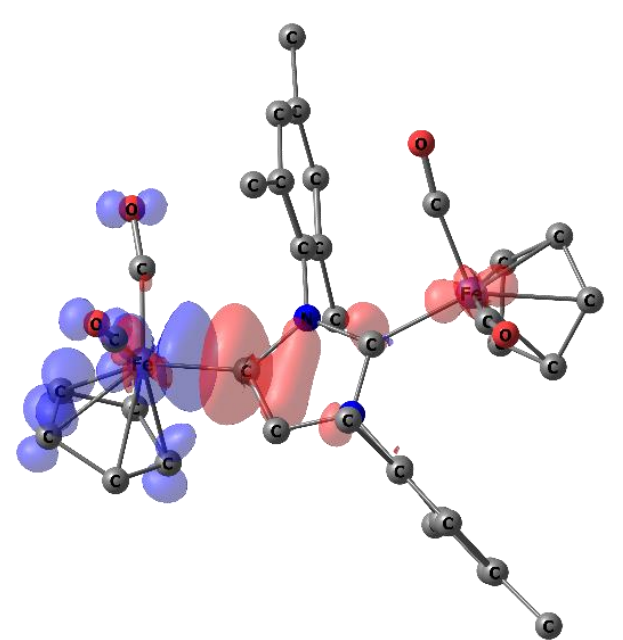

NOCV1, $E=-71.5(\sigma C: \rightarrow M)$

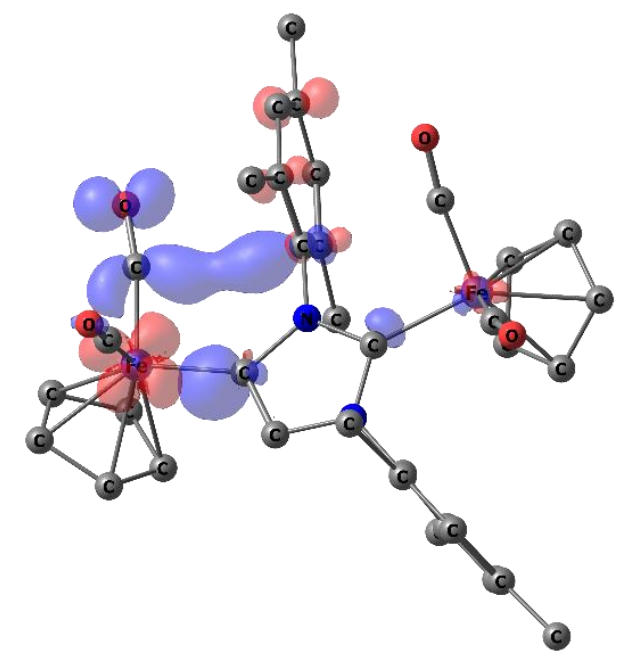

NOCV3, $E=-5.1\left(\pi(C=C) \rightarrow \pi^{*}(C O)\right)$

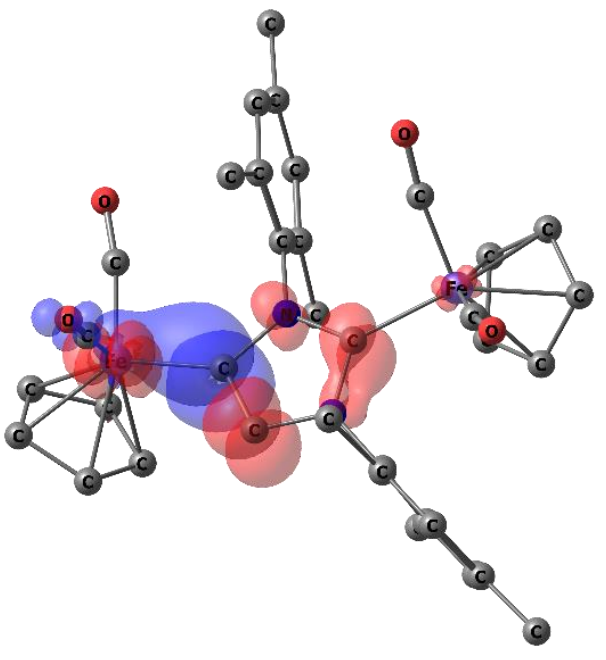

NOCV2, $E=-8.7\left(\pi_{\perp} M \rightarrow C:\right)$

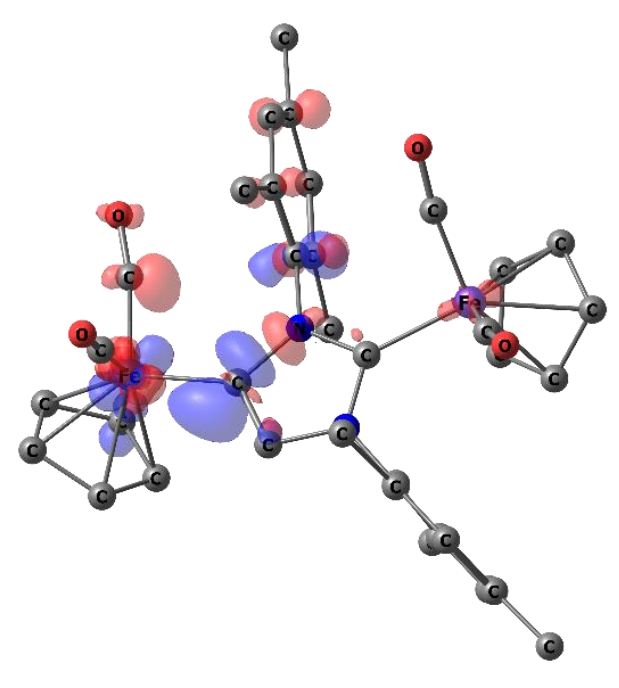

NOCV4, $E=-3.2\left(\pi_{\|} M \rightarrow C:\right)$

Figure S33. NOCV deformation electron density for $\mathbf{F e}-a \mathrm{NHC}$ bond in complex $\mathbf{5 \mathbf { a } ^ { + }}$ (isosurface at 0.003 and 0.001 a.u. for NOCV1 and NOCV2-4, respectively) and associated energies in $\mathrm{kcal} \mathrm{mol}{ }^{-1}$. Red areas correspond to the depletion and blue to the concentration of electron density. Hydrogen atoms of aryls are omitted for clarity. 


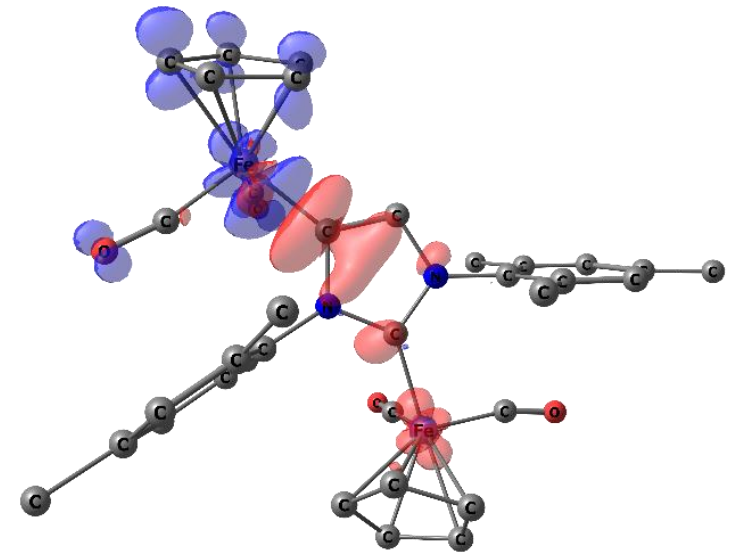

NoCV1, $E=-71.3(\sigma \mathrm{C}: \rightarrow \mathrm{M})$

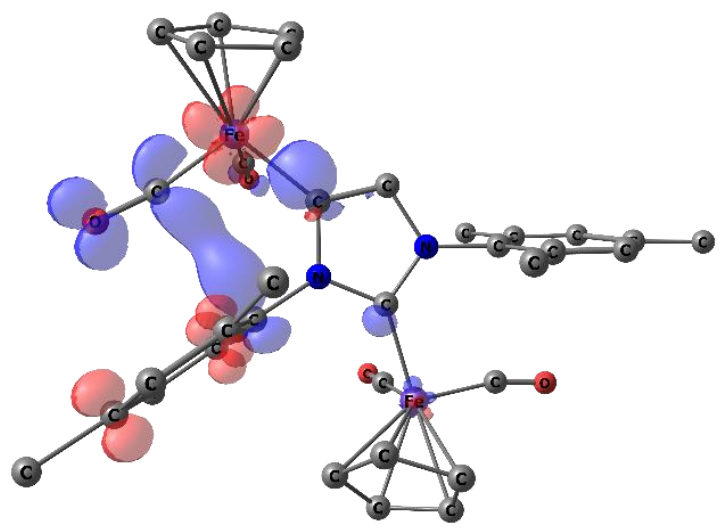

NOCV3, $E=-5.0\left(\pi(C=C) \rightarrow \pi^{*}(C O)\right)$

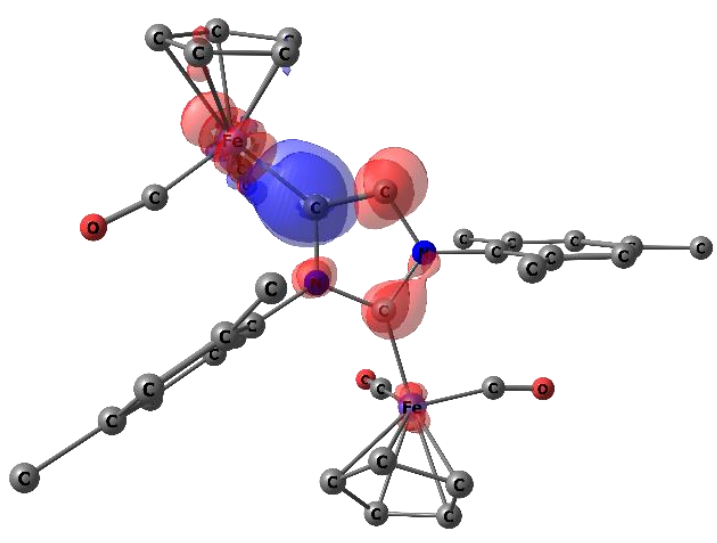

NOCV2, $E=-9.0\left(\pi_{\perp} M \rightarrow C:\right)$

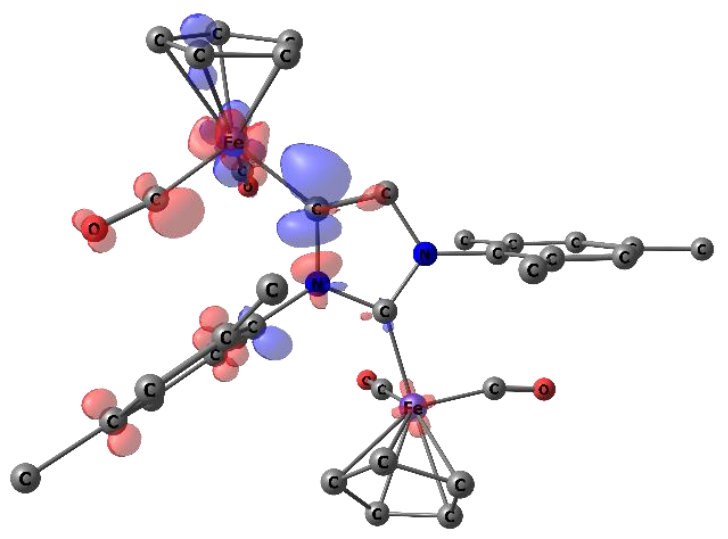

NOCV4, $E=-3.4\left(\pi_{\|} M \rightarrow C:\right)$

Figure S34. NOCV deformation electron density for Fe-aNHC bond in complex $\mathbf{5} \mathbf{b}^{+}$(isosurface at 0.003 and 0.001 a.u. for NOCV1 and NOCV2-4, respectively) and associated energies in $\mathrm{kcal} \mathrm{mol}^{-1}$. Red areas correspond to the depletion and blue to the concentration of electron density. Hydrogen atoms of aryls are omitted for clarity. 